\title{
An Open Source Inertial Sensor Network with Bluetooth Smart
}

by

Hao Yan

A thesis submitted in conformity with the requirements

for the degree of Master of Applied Science

Department of Electrical and Computer Engineering

University of Toronto

(C) Copyright by Hao Yan, 2015 


\title{
An Open Source Inertial Sensor Network with Bluetooth Smart
}

\author{
Hao Yan \\ Master of Applied Science \\ Department of Electrical and Computer Engineering \\ University of Toronto
}

2014

\section{Abstract}

In this thesis, an open source inertial sensor network with Bluetooth Smart connection is presented. The network has multiple sensor nodes connecting to a consumer electronic device with Bluetooth Smart. Each sensor node contains the following components: (a) an inertial sensor measuring acceleration, angular velocity, and magnetic field with good accuracy; (b) a microcontroller with capacity to handle real-time floating number calculations; (c) a Bluetooth Smart module broadcasting the data with low power consumption. The sensor nodes are designed to be small, allowing the users to wear them conveniently. For demonstration, a basic Personal Navigation System is developed using 4 of these sensor nodes and an Android smartphone. The experiments show that the sensor nodes could output accurate results with small noises when at rest or in slow motion. The example Personal Navigation System could measure total distance walked by a pedestrian with less than $10 \%$ error. 


\section{Acknowledgments}

I wish to express my gratitude to my supervisor, Professor David A. Johns, for his patient guidance and encouragement throughout my time as his student. Professor Johns has offered me inspiring advices from choosing the thesis subject to writing the thesis. I would not be able to overcome some obstacles during the project without his unreserved assistance.

I would like to thank Dr. Chuhong Fei and Dr. Zhiyun Lin for devoting time to read my manuscript and providing me with significant and constructive suggestions. Their valuable input helps me enormously.

Thanks to Fengmin Gong from Zhejiang University for guiding me on PCB board design and manufacture. Without his kind assistance, I would not be able to finish the prototype board in time.

Finally I would like to thank my family for their continuous support since my undergraduate years. I would like to thank my father especially for discussions on debugging issues of the project. 


\section{Table of Contents}

$\begin{array}{ll}\text { Acknowledgments } & \text { iii }\end{array}$

Table of Contents $\quad$ iv

List of Tables

List of Figures

List of Acronyms $\quad$ xi

Chapter 1: Introduction $\quad 1$

1.1 Introduction to inertial sensor networks 1

1.2 Applications of inertial sensor networks 2

$\begin{array}{lll}1.3 & \text { Motivation } & 4\end{array}$

1.4 Thesis outline $\quad 5$

$\begin{array}{ll}\text { Chapter 2: Background } & 6\end{array}$

$\begin{array}{lll}2.1 & \text { Inertial measurement units } & 6\end{array}$

$\begin{array}{lll}2.2 & \text { Wireless communication protocols } & 7\end{array}$

2.2.1 Wi-Fi and Wi-Fi Direct $\quad 8$

2.2.2 ZigBee $\quad 8$

2.2.3 Bluetooth 9

2.2.4 Bluetooth Smart 9

$\begin{array}{lll}2.3 & \text { Wearable body sensor networks } & 10\end{array}$ 
4.1 How to determine the current step length

4.2 How to differentiate between steps

4.3 Android app design

Chapter 5: Experiments and Results

5.1 Performance of individual sensor node

5.1.1 Data accuracy at rest

5.1.2 Data accuracy while moving

5.1.3 Data rate limitation for BLE

5.1.4 Battery Life

5.2 Performance of inertial sensor network 66

5.3 Results of MotionTracker PNS

Chapter 6: Conclusion and Future Work

6.1 Conclusions

6.2 Future work on the inertial sensor network

Reference

Appendices

A. Cost of components for a sensor node

B. Source code for sensor node software

C. Source code for MotionTracker app 
D. Schematics and PCB design files for the sensor node board 


\section{List of Tables}

Table 1: Possible applications of inertial sensor networks

Table 2: Current consumption of major components in working and sleeping modes

Table 3: Unit price of major components of the latest purchase from Digikey, before tax, in Canadian Dollar

Table 4: Selected specifications for TM4C123GH6PM

Table 5: Comparison of sensor specifications between MPU9150 and 3DM-GX3

Table 6: Commands, Events, and Responses for sensor node to have basic functions

Table 7: The state machine for MotionTracker

Table 8: The acceleration data for Sensor Node 2 when at rest on desk surface

Table 9: The gyroscope data for Sensor Node 2 when at rest on desk surface

Table 10: The Magnetometer results for Sensor Node 2 when at rest on desk surface

Table 11: The Euler Angle results for Sensor Node 2 when at rest on desk surface

Table 12: Comparison between the angle measured by the sensor node and the pictured angle on corresponding video frame (unit: degree)

Table 13: Periods between transmissions at receiving end for different data transmission rate 64

Table 14: Transmission rate measured for 4 sensor nodes at receiving end

Table 15: MotionTracker experiment results with step by step measurements.

Table 16: Complete list of component costs of a sensor node 


\section{List of Figures}

Figure 1: The IMU's reference frame, assuming it is a rigid body.

Figure 2: A typical personal navigation system based on Pedestrian Dead Reckoning (PDR). 13

Figure 3: The block diagram of a sensor node in our design. The red line represents 3.3V power lines; the green line represents I2C channel; the blue line represents UART channel. The arrow represents the flow of data and control.

Figure 4: The top view of a sensor node board

Figure 5: Code snippet for configuring UART channels

Figure 6: Code Snippet for preparing the BLE113 module for connection

Figure 7: Code Snippet for the MPU9150 initialization sequence

Figure 8: The figure above shows the data flow from sensor to the Bluetooth module.

Figure 9: Code snippet for some of the handling methods of BLE events and responses

Figure 10: Content of hardware.xml for the sensor node

Figure 11: The general architecture of the MotionTracker system. The Main Body Sensor SMB1

provides headings data. Each limb sensor reports its orientation in Euler angles.

Figure 12: A pedestrian stepping with his right leg

Figure 13: Denotations of Euler angles and relative displacements along primary direction, viewed from the side of pedestrian

Figure 14: Denotation of Euler angles, viewed from the back of pedestrian

Figure 15: The accelerometer results for Sensor Node 2 when at rest on desk surface

Figure 16: The gyroscope data for Sensor Node 2 when at rest on desk surface 
Figure 17: The Euler angle data for Sensor Node 2 when at rest on desk surface

Figure 18: Euler angle in $\mathrm{X}$ axis (roll, the main axis of motion) for robotic arm to move between 90 and 45 degrees

Figure 19: Euler angle in Y axis (pitch, off-motion axis)

Figure 20: Euler angle in $\mathrm{Z}$ axis (yaw, off-motion axis) 


\section{List of Acronyms}

- ADC - Analog-to-Digital Converter

- BSN - Body Sensor Network

- BLE - Bluetooth Low Energy

- CAN - Controller Area Network

- DCM - Direction Cosine Matrix

- DMIPS - Dhrystone Million Instruction Per Second

- EEPROM - Electrically Erasable Programmable Read-Only Memory

- FPU - Floating-Point Unit

- GAP - Generic Access Profile

- GATT - General Attributes Profile

- GPIO - General-Purpose Input/Output

- GPS - Global Positioning System

- I2C - Inter-Integrated Circuit

- ICDI - In-Circuit Debug Interface

- IMU - Inertial Measurement Unit

- LAN - Local Area Network

- MEMS - Micro-Electro-Mechanical systems

- NVIC - Nested Vectored Interrupt Controller 
- $\quad$ PDR - Pedestrian Dead Reckoning

- PNS - Personal Navigation System

- PLL - Phase-Locked Loop

- PWM - Pulse-Width Modulation

- SINS - Ship's Inertial Navigation System

- SSI - Synchronous Serial Interface

- UART - Universal Asynchronous Receivers/Transmitter

- UUID - Universally Unique Identifier

- WSN - Wireless Sensor Network

- ZUPT - Zero-velocity Updates 


\section{Chapter 1: Introduction}

This thesis presents a network of wearable inertial sensors with Bluetooth Smart connections aiming for consumer applications development. The results show that, with basic low-cost MEMS sensors, the system is able to collect measurements from 4 separate sensor nodes at $40 \mathrm{~Hz}$, which is sufficient for many consumer applications.

This chapter has 4 sections. Section 1.1 introduces the basics of inertial sensor networks. Section 1.2 lists some of the possible applications of inertial sensor networks. Section 1.3 explains the motivations behind this project. Section 1.4 outlines the organizing structure of this thesis.

\subsection{Introduction to inertial sensor networks}

An inertial sensor network is a group of sensor nodes containing Inertial Measurement Units (IMUs) which can measure acceleration, angular velocity, and magnetic field at various locations. These inertial sensor nodes can be worn on the human body. An inertial sensor network can sometimes be part of a Body Sensor Network (BSN) [1], or used with video camera for action capture [2]; it can also form the backbone of a Personal Navigation System (PNS) [3]. Inertial sensor networks can be used to measure muscle activities, recognize gestures, and evaluate actions when placed at key locations on a human body. They have seen many applications in personal navigation, fitness and health treatments, sports activities and dancing, gaming, movies and anime production. Due to the essential differences in applications, these inertial sensor networks are implemented disparately, specialized in their own tasks to achieve maximum performance. Such difference is fine for industry or professional use, since performance outweighs compatibility in most cases. However, to enter the consumer market, inertial sensor networks need to be compatible with consumer electronic devices: smartphones, tablets, and PCs. In addition, a single inertial sensor network should be able to perform various tasks, allowing many applications to use the same hardware.

Typically a sensor node constitutes an IMU, a microcontroller for data processing, a power source, and a wireless transmission module. The microcontroller drives the IMU to 
collect data at a designated frequency. The microcontroller is then able to apply simple filtering to the inertial data reported by the IMU, removing errors and improving accuracy. Finally, the microcontroller interacts with the wireless communication module for transmitting data and receiving commands.

Wireless inertial sensor networks use various methods to transmit data. The wireless transmission protocol of such a sensor network is normally designed to maximize battery life while achieving a required data rate for its specific task. Other factors, such as range and latency, are taken into account but often considered less important. In some cases, the requirements for high data rate and low power consumption force developers to implement custom radio protocols specifically designed for the tasks to achieve optimal

performance [4]. There are a few wireless transmission protocols that have wide coverage in consumer electronic devices: Wi-Fi, ZigBee, Bluetooth, and Bluetooth Smart. Inertial sensor networks have to support at least one of them to be able to communicate with most personal computing devices.

All the data from sensor nodes are gathered at a remote processing device. By analyzing the data, either real-time or afterwards, the device is able to calculate interesting results and make useful judgments. In some cases, these results are used to supplement measurements of other sensing devices, such as GPS or a video camera. Some applications require synchronized data; hence the remote processing device may also arbitrate the timings for data collection in all sensor nodes. Most consumer electronic devices, such as smartphone and tablets, have more than enough computing power to process the sensor data for inertial sensor network applications. The additional sensors often found in such devices could also be incorporated into these applications.

\subsection{Applications of inertial sensor networks}

One important application of inertial sensor network is PNS. PNS can provide navigation in places where GPS signals are weak or unavailable [5]. In case the GPS signal is not strong enough to determine the location, the PNS takes over to continue navigation. Some indoor navigation systems incorporate the PNS with computer vision 
for better accuracy. Recently, new developments in PNS have made it possible to be a standalone navigation system inside buildings [6] [3] [7].

The inertial sensor network has also seen many applications in the video game industry, robotics, and medical field. Table 1 shows a list of the possible applications.

Table 1: Possible applications of inertial sensor networks

\begin{tabular}{|c|c|}
\hline Environment and Context & Functions \\
\hline $\begin{array}{c}\text { Inside large public buildings such as } \\
\text { shopping malls, hospitals, schools, and } \\
\text { office towers }\end{array}$ & Indoor navigation [6] \\
\hline Jungles, forests, and underground caves & $\begin{array}{l}\text { Support GPS for consistent navigation } \\
\qquad[5]\end{array}$ \\
\hline $\begin{array}{c}\text { Gyms, laboratories, physical activity } \\
\text { researches }\end{array}$ & $\begin{array}{l}\text { Plot and analyze user actions for fitness, } \\
\text { performance, and sports [4] }\end{array}$ \\
\hline Hospitals, home & $\begin{array}{l}\text { Measure patient exercises for } \\
\text { rehabilitation [1] }\end{array}$ \\
\hline $\begin{array}{c}\text { Anime, Computer Graphics, Movies, } \\
\text { Computer Games }\end{array}$ & Action and motion capture [2] \\
\hline $\begin{array}{l}\text { Used with robots in tunnels, debris, and } \\
\text { deep seas }\end{array}$ & $\begin{array}{l}\text { Motion control, navigation and action } \\
\text { feedback for robots }\end{array}$ \\
\hline
\end{tabular}

Most of these applications require users to wear the sensors and use a separate device for data compilation and analysis. While inertial sensor networks have already seen many applications in industry, scientific research, medical treatment, and military actions, they generally remain out of touch for common people. The primary reasons are the high costs and poor performances. The physical size and battery life of sensor nodes are also limitations for inertial sensor networks to enter the consumer market. 


\subsection{Motivation}

Most inertial sensor networks are custom built to perform a few very specific tasks. They often use highly specialized sensors and data transmission methods to achieve the desired accuracy, data rate, and battery life. Although these systems can perform reasonably well for their tasks, they can hardly be useful for other purposes without extensive calibration and modification. For example, it is almost as difficult to convert a body sensor network that recognizes gestures to an indoor navigation system as to build a new system from scratch. In addition, high-precision sensors, which usually cost much more than common consumer electronic devices, are used to achieve best performance. As a result, there are very few consumer-orientated applications based on inertial sensor networks.

This thesis attempts to resolve some of the problems stated above by providing an open source inertial sensor network with low hardware costs for research and developments. The goal of this project is to demonstrate that a common architecture can output inertial data with reasonable accuracy and frequency for many applications with only low cost MEMS sensors. The inertial sensor network is designed to be used with personal computing devices: smartphones, tablets, and PCs. Applications that perform different tasks can be developed on these devices, using the same inertial sensor network hardware.

To achieve the goal, the system is built with low cost components, with total component cost of around CAD $\$ 70$ for a sensor node. Each sensor node has small physical size, allowing them to be worn conveniently by users. Every sensor node has real-time floating point processing capability for sensor fusion and signal filtering, reducing the bandwidth needed by transmitting processed data rather than raw data. A smartphone can collect data from 4 sensor nodes of the system at the same time, each producing 40 sets of readings per second.

Ultimately the proposed system is intended to make potential research and development on consumer-oriented applications of inertial sensor networks easier. A 
simple PNS is developed as an example, achieving measurements with errors less than $10 \%$ of total distance in experiments.

\subsection{Thesis outline}

The rest of the thesis is organized into 5 chapters:

- Chapter 2 introduces previous works related to inertial sensor networks and their applications.

- Chapter 3 describes the hardware and software implementations of each sensor node, covering the microcontroller programming and the Bluetooth configurations.

- Chapter 4 describes the PNS example centering around two questions: how to accurately estimate the step length from angles; how to determine steps and sum up their step lengths to produce the distance correctly.

- Chapter 5 explains the experiment methods and presents the results in details.

- Chapter 6 concludes the thesis by discussing the limits of our work and potential future developments. 


\section{Chapter 2: Background}

This chapter covers the background of inertial sensor networks. The first two sections, 2.1 and 2.2, introduce Inertial Measurement Units (IMUs) and wireless communication protocols. The remaining two sections, 2.3 and 2.4, focus on the two most important applications of inertial sensor networks: Body Sensor Network (BSN) and Personal Navigation System (PNS). This chapter also describes some recent research in these fields.

\subsection{Inertial measurement units}

An inertial sensor network includes many sensor nodes with Inertial Measurement Units (IMUs). IMUs are accelerometers, gyroscopes, and magnetometers, or any combinations of these 3 types of sensors. An IMU measures the current rate of acceleration using an accelerometer, and detects the changes in rotational angles such as roll, pitch, and yaw with a gyroscope. The magnetometer is sometimes used for initializing the orientation, and calibrating for drifts caused by integrating the results from a gyroscope.

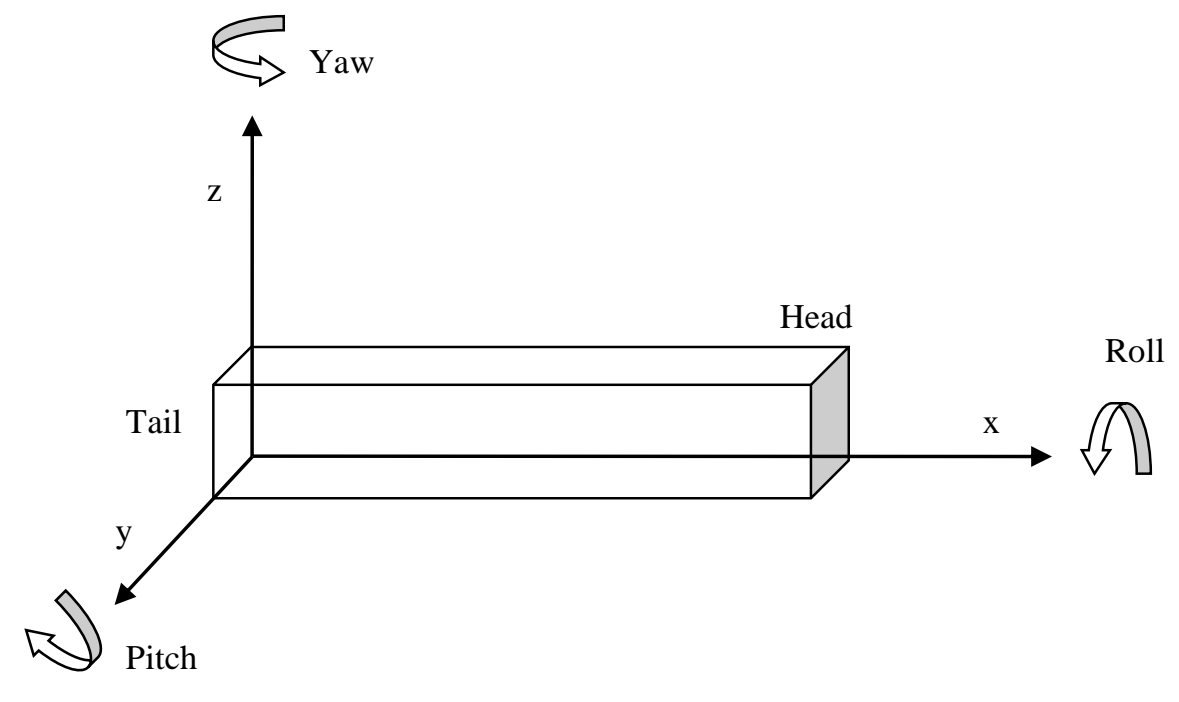

Figure 1: The IMU's reference frame, assuming it is a rigid body. 
Figure 1 shows the frame of reference of an IMU. For this IMU, the accelerometer measures the acceleration in 3 directions $(\mathrm{x}, \mathrm{y}, \mathrm{z})$ and the gyroscope normally expresses angular velocity in (roll, pitch, yaw). The pitch angle represents the head up and down comparing to the horizontal surface. The roll angle represents the clockwise and counterclockwise rotation around the axis from head to tail. The yaw angle represents the heading on the horizontal surface. These angles are around their corresponding axes: roll for $\mathrm{x}$, pitch for $\mathrm{y}$, and yaw for $\mathrm{z}$. They generally use right hand rule to determine the positive rotational direction.

IMUs' most important application is navigation. Based on the accelerometer and gyroscope readings, it is possible to use integration to calculate the velocity, displacement, and orientation of the objects they attach to. However the integration process is prone to errors as the static errors in acceleration and angular velocity could accumulate to a large amount over time. Various studies have centered on limiting this error growth by establishing a real-time error estimation model that could adapt to different situations. As a simple example, to estimate the current orientation of a sensor node with reasonable accuracy, a complimentary filter could be applied on the orientation angles produced by accelerometer and gyroscope readings, since results from accelerometer are more accurate when the sensor node is near static while the results from the gyroscope are more accurate when the sensor node is rotating.

Many IMUs have an internal clock with relatively low accuracy. This clock dictates the timings to read measurements, and often leads to small errors in measurement frequency. Most of the time the errors are negligible, but some applications require better precision in the timing of measurements. In these cases, a more accurate external crystal could be used to drive the IMU.

\subsection{Wireless communication protocols}

An inertial sensor network is a type of Wireless Sensor Networks (WSNs), with all sensor nodes containing inertial sensors. There are various wireless communication protocols for WSNs, such as Wi-Fi, ZigBee, Bluetooth, and Bluetooth Smart, each aiming for different applications. In general, the target of wireless communication 
protocol is to achieve high throughput, low latency, and long battery life. These protocols may not always satisfy the needs of the application, so sometimes custom radio protocols are needed. In this section, some protocols commonly found in WSNs are reviewed.

\subsubsection{Wi-Fi and Wi-Fi Direct}

$\mathrm{Wi}-\mathrm{Fi}$ is the most widely used wireless communication protocol for Internet access at home, workspace, and public hotspots. Wi-Fi is able to transmit large amounts of data at a fast rate, at the cost of high power consumption and low efficiency (large overheads). Wi-Fi requires all the data transmitters and the data receivers to connect to the Internet or a Local Area Network (LAN) via established access points. Hence a wireless sensor network based on Wi-Fi cannot move beyond the coverage of $\mathrm{Wi}-\mathrm{Fi}$ access points. Thus $\mathrm{Wi}-\mathrm{Fi}$ is often considered to be less desirable than other protocols for a WSN, unless there are large amounts of data that needs continuous streaming from the sensor nodes to the data receiver.

To avoid the complications of connecting to fixed access points, the Wi-Fi devices could connect directly with each other using Wi-Fi Direct technology [8]. Wi-Fi Direct allows sensors and data receiver to form a group, and data can be transmitted between group members. It essentially makes one device as the access point and all other devices connect to it. Wi-Fi Direct allows the wireless sensor network to function anywhere, as long as all the sensor nodes are within range of the data receiver. However, other than this, the technology still experiences the same problems as Wi-Fi.

\subsubsection{ZigBee}

ZigBee is almost the opposite of Wi-Fi. It is suitable for applications that require relatively low data rate and long battery life. ZigBee is based on the IEEE 802.15.4 standard, with 3 different data rates of $250 \mathrm{Kbit} / \mathrm{s}, 40 \mathrm{Kbit} / \mathrm{s}$, and $20 \mathrm{Kbit} / \mathrm{s}$ [9]. ZigBee has a transmission range comparable to $\mathrm{Wi}-\mathrm{Fi}$ : 1-100 meters. One of the most important features for ZigBee is its scalability. ZigBee can support a large number of sensor nodes with low latency, using star and mesh networks. The mesh network also allows ZigBee to extend its reach beyond the limited transmission range by passing the data from one sensor node to another. In addition, ZigBee employs Direct Sequence Spread Spectrum. 
The data being transmitted is multiplied to a sequence of 1 and -1 values at the transmitting end, and is reconstructed using the same sequence at the receiving end. This enables a ZigBee sensor network to remain quiescent for long periods of time without close synchronization, thus conserving the battery life even more. The greatest limitation for ZigBee is that currently many smartphones and tablets do not support the protocol. A WSN built with ZigBee is unable to work with most consumer electronic devices directly, preventing it from entering the consumer market.

\subsubsection{Bluetooth}

Bluetooth technology is designed to work with low power consumption [10]. Its most common class 2 radios use only $2.5 \mathrm{~mW}$ of power when working. However this low energy cost means a short range for transmission, the same class 2 radios could only reach a maximum of 10 meters, much shorter than other wireless communication protocols. The theoretical data throughput for a Bluetooth 3.0 (or newer version) device is near $24 \mathrm{Mbit} / \mathrm{s}$, better than ZigBee.

All Bluetooth technology, including Bluetooth Smart, pairs two devices when they make a connection. Data can be transmitted between a pair of devices. In classic Bluetooth, each device can be paired with at most 7 other devices at the same time. This limits the maximum number of nodes for a WSN built with Bluetooth technology, and force the network structure to be a star network. Furthermore, Bluetooth technology's main purpose is to transfer data and voice at the same time, which is irrelevant for a WSN.

\subsubsection{Bluetooth Smart}

Bluetooth Smart, also called Bluetooth Low Energy (BLE), is the new generation of Bluetooth technology specified in the Bluetooth 4.0 standard, which is more intelligent (hence: Bluetooth Smart) about managing connections, especially when it comes to conserving energy. The low power consumption of Bluetooth Smart could allow a coin battery to power a BLE device for years. That is because BLE device is kept in sleep mode most of the time until a connection is made, and each connection may only last a few milliseconds. BLE uses the same $2.4 \mathrm{GHz}$ band as classic Bluetooth. BLE has a data 
rate of $1 \mathrm{Mbit} / \mathrm{s}$, less than the classical Bluetooth, but its latency, transmission speed, and connection speed are much better. BLE also provides a possible range for more than 100 meters with increased modulation index. Unlike classic Bluetooth, Bluetooth Smart is not limited to have a maximum of 7 slaves per master. The actual number of slave devices supported depends on the implementation and available memory of the master device. This allows inertial sensor networks with Bluetooth Smart to be scalable.

The actual power consumption of BLE varies from $0.01 \mathrm{~W}$ to $0.5 \mathrm{~W}$, depending on the data transmission frequency. To maximize the battery life, it is best to use Bluetooth Smart for tasks with a small amount of data transmission and light duty cycle. This is usually not the case for an inertial sensor network, as a continuous flow of inertial data is needed. However, even in this case, BLE still consumes less power than other alternative wireless communication technologies commonly found in consumer electronic devices. There are also a few suitable applications, such as a heart rate monitor, that can fully utilize its power-saving ability. The only drawback for Bluetooth Smart is the limited throughput, but processing inertial data locally at each sensor node can alleviate the problem, since only the relevant results need to be transmitted.

Currently, Bluetooth Smart is supported by many consumer electronic devices. It is chosen for our system because of its low power consumption and wide availability.

\subsection{Wearable body sensor networks}

The small size and the capabilities to produce real-time results make inertial sensors ideal for wearable BSNs. A Body Sensor Network, also called Body Area Network (BAN), is composed of wearable computing devices that are often connected wirelessly. BSNs are initially developed for health monitoring, such as periodically reporting realtime medical records of patients. These BSNs often integrate a number of physiological sensors to detect certain medical conditions. The sensors collect various physiological data of human body and send them wirelessly to a data processing device for analysis.

In recent years, BSNs have seen many applications outside the medical field. In movie and animation production, BSNs with inertial sensors are used for action capture, 
often to supplement video cameras. This is because sometimes video cameras are unable to provide enough information for human actions, especially when the actions are fast and minimal in scale, making them difficult to be captured visually. IMUs can measure at a faster rate than most video cameras and detect even the smallest motion. In other cases, the body motion data collected by BSNs are used for analysis, helping athletes and performers to improve their training quality. Analysts can look at the very details to understand how a subject performs the actions, and point out possible improvements the subject could use.

The errors from integration are a major problem for inertial sensor networks. Hence, in many BSNs, other sensors, such as acoustic sensors that can measure distance between sensors [11], are used to overcome this problem. Otherwise, algorithms and systems are conceived to avoid using integration altogether. Ronit Slyper and Jessica K. Hodgins [2] describe a performance animation system using an inertial sensor network. They place sensors on a tight shirt and let a person wear it to do all kinds of actions. They do not use acceleration for integration; instead they compare the sensor readings with an established acceleration database of actions, using the closest match to animate an avatar. The database of accelerations is computed from normal video capture. As expected, the system performs well for repeatable and easily recognizable actions; for more random actions, it often fails to find a good match in the database.

Some studies only collect, analyze and utilize raw sensor data: acceleration, angular speed, and magnetic field. Ryan Alyward and Joseph A. Paradiso build a wearable BSN that can measure, analyze the actions of dancers and transform them to musical parameters real-time, using only raw sensor data [4]. To account for the need to describe motions in dance, they design the system to have huge bandwidth, allowing a large set of data to be transmitted at a high frequency. At the same time, the sensor nodes have to be kept small enough so as not to disrupt the movements of performers and still have long enough battery life. Such extreme requirements force them to design a custom radio protocol that can achieve high data rate as well as low power consumption. By analyzing the magnitude of peak acceleration and angular velocity, plus the global activity level of 
all sensors, they are able to generate interactive music corresponding to these measurements.

Similar action based inertial data analysis could also be applied back to the medical field, where patients suffering from injuries could use it for recovery. A service based on BSNs is proposed to help patients in post-surgical knee rehabilitation [1]. The rehabilitation often involves training in a hospital gym under the supervision of a physiotherapist. This is to ensure the patients execute the movements correctly and adjust the exercises level according to the level of recovery. This service could automatically evaluate the conditions of patients, allowing the physiotherapist to control their exercises remotely. The experimental results show that the system is suitable to evaluate patients' performance for a few selected exercises.

\subsection{Personal navigation systems}

Common personal navigation systems often employ either Pedestrian Dead Reckoning (PDR) method or Ship's Inertial Navigation System (SINS) [12] method. SINS method relies on accurate sensor readings to provide meaningful navigation, as any errors would cause the results to diverge quickly. On the other hand, PDR systems separate the navigation algorithm into the location calculation and the step calculation. To calculate a step, normal PDR systems integrate the accelerometer and gyroscope readings to get the distance and direction for a step. With an initial position specified, the consecutive steps with length and direction calculated are able to locate the pedestrians on the move.

Regardless of SINS or PDR, PNS often applies the Zero-Velocity updates (ZUPT) method to prevent error accumulation over time. ZUPT was based on the fact that when a pedestrian walks, there is a stance phase in which one foot is on the ground and the sensor on that foot must be at rest. During this phase the accumulated errors from integration can be reset so the errors do not inflate indefinitely. This algorithm, nonetheless, still requires accelerometers to be substantially accurate so errors do not accumulate to a significant amount during a step's time. More recent researches have taken this method one step further. In addition to resetting the velocity to 0 , new systems 
employ an Extended Kalman Filter to feedback the current error in speed, adjusting the error correction mechanism for next step [13]. Over time, the Extended Kalman Filter can produce an error correction matrix tailored for the walking pattern of a user, achieving maximum accuracy. The general block diagram of such systems is shown in Figure 2.

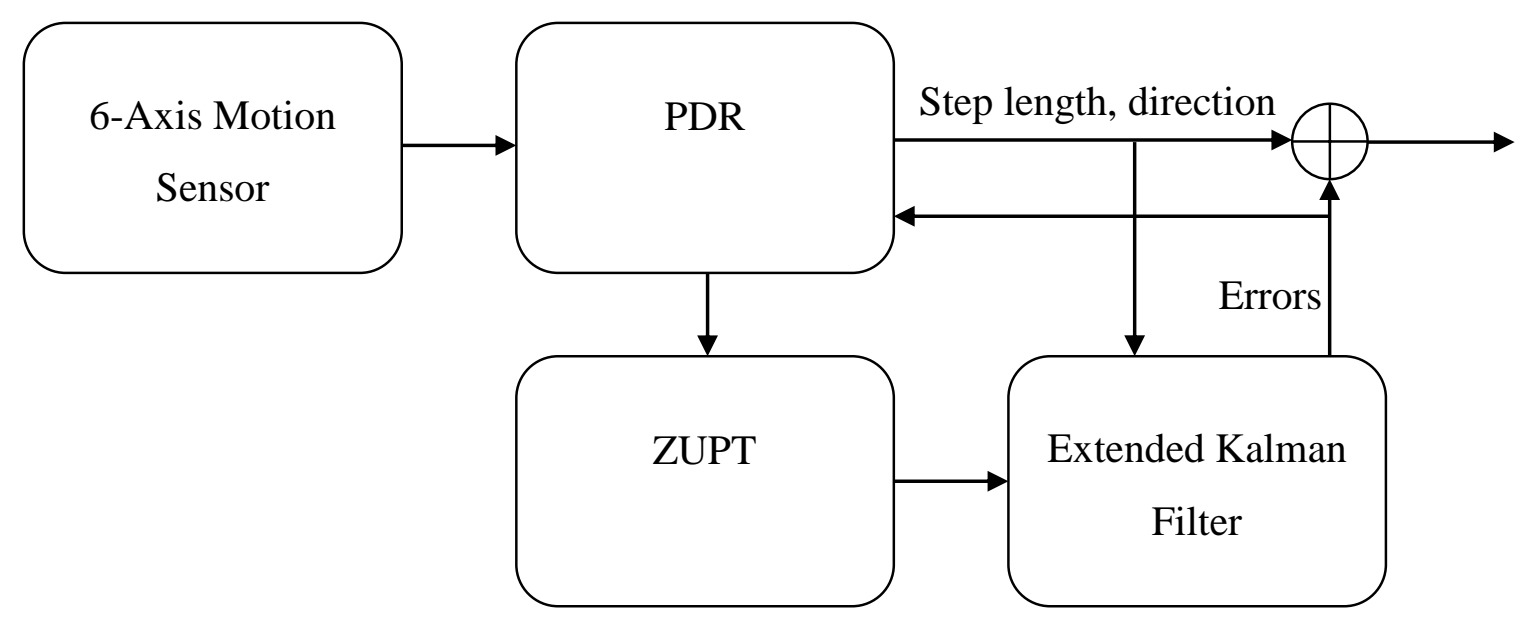

\section{Figure 2: A typical personal navigation system based on Pedestrian Dead Reckoning (PDR).}

Although in theory the sensors can be attached to any part of the body that can detect the biomechanics of a step, a shoe-mounted sensor is most intuitive, and therefore used in most PNSs [14]. Eric Foxlin [13] presents such a system as early as in 2005. Recent researches report an error of less than $2 \%$ of the total distance [15]. Similar results are also achieved with systems using the SINS method [7].

One drawback of shoe-mounted PNSs is that with different choices of the sensors, setup configurations and experiment environments, the results may vary greatly from one system to another. The errors could become much larger than normal in difficult terrain [16] or in places with magnetic disturbances. Accelerometers that can measure the shocks on the shoes often lack in the precision comparing to those with smaller range.

Juan Carlos Alvarez et al. present a waist-worn inertial navigation system which also uses human bipedal pattern to reduce position errors [3]. The system is based on the fact that when both feet are on the ground, the vertical velocity of a waist-worn sensor is 
expected to be 0 . Thus the system can translate heel strike biomechanics to accelerations on body at waist to estimate periods of zero velocity. Using ZUPT, the system can calculate step length, heading, and track the overall pedestrian movement with less than $2 \%$ error.

However ZUPT cannot help estimate errors in headings. By applying constraints to a shoe-mounted PNS, more accurate results can be achieved. Considering the environment inside a building, Abdulrahim, K. et al. make the following assumptions for a pedestrian: the heading is always along the hallway; the heading remains constant at stop; the elevation doesn't change anywhere else except on staircases [6]. These assumptions enabled constraints on the heading, the heading drifts at rest, and the error growths on height. With these constraints in place and knowledge of the layout of the building, a position error of mere 4.62 meter is achieved for a total distance of $1557 \mathrm{~m}$. 


\section{Chapter 3: Implementation of Sensor Node}

This chapter describes the hardware and software implementation of a sensor node. First, in section 3.1, the overall architecture of a sensor node is outlined. Then the hardware and electrical implementation of a sensor node board is presented in section 3.2. In section 3.3, the microcontroller software of a sensor node is described in details. Finally, section 3.4 focuses on the configurations of the Bluetooth Smart module.

\subsection{Overall architecture of a sensor node}

For the inertial sensor network presented in this thesis, all the sensor nodes are the same for simplicity and compatibility with applications. A sensor node measures acceleration, angular velocity, and magnetic field for all axes / directions, providing allaround inertial data. In addition, the sensor node has the computing power to process these measurements real-time to filter noises and produce sensor fusion results. Finally, the sensor node transmits these results wirelessly to a consumer electronic device for analysis.

A PCB board is designed to meet the above requirements to implement the sensor

node. A single piece sensor node hardware is mainly composed of a controlling microprocessor, TM4C123GH6PM, a BLE module, BLE113, and a 9-axis MEMS motion sensor, MPU9150. In addition to these main components, a tri-color LED is present on the board; it can be used to indicate different states of the sensor node. There are also three buttons on the board, one is used for resetting the board, while the others are free for programming. Figure 4 shows the top view of a finished sensor node.

The architecture of such a sensor node is shown in Figure 3. The sensor node is controlled by the microcontroller, which handles interactions with the motion sensor and forward results to the BLE module. At power-on, the microcontroller executes steps to initialize the motion sensor and BLE module, preparing for data collection and transmission. Once the initialization is complete, the microcontroller enters an infinite loop, continuously reading data from MPU9150 and processing them. On the other hand, the BLE module does nothing until a remote BLE device is connected. As the BLE113 
module alerts the microcontroller of the connection event, the microcontroller starts to update the General Attributes Profile (GATT) server in BLE module with the latest sensor data. The GATT server can send the data to the client in the remote BLE device.

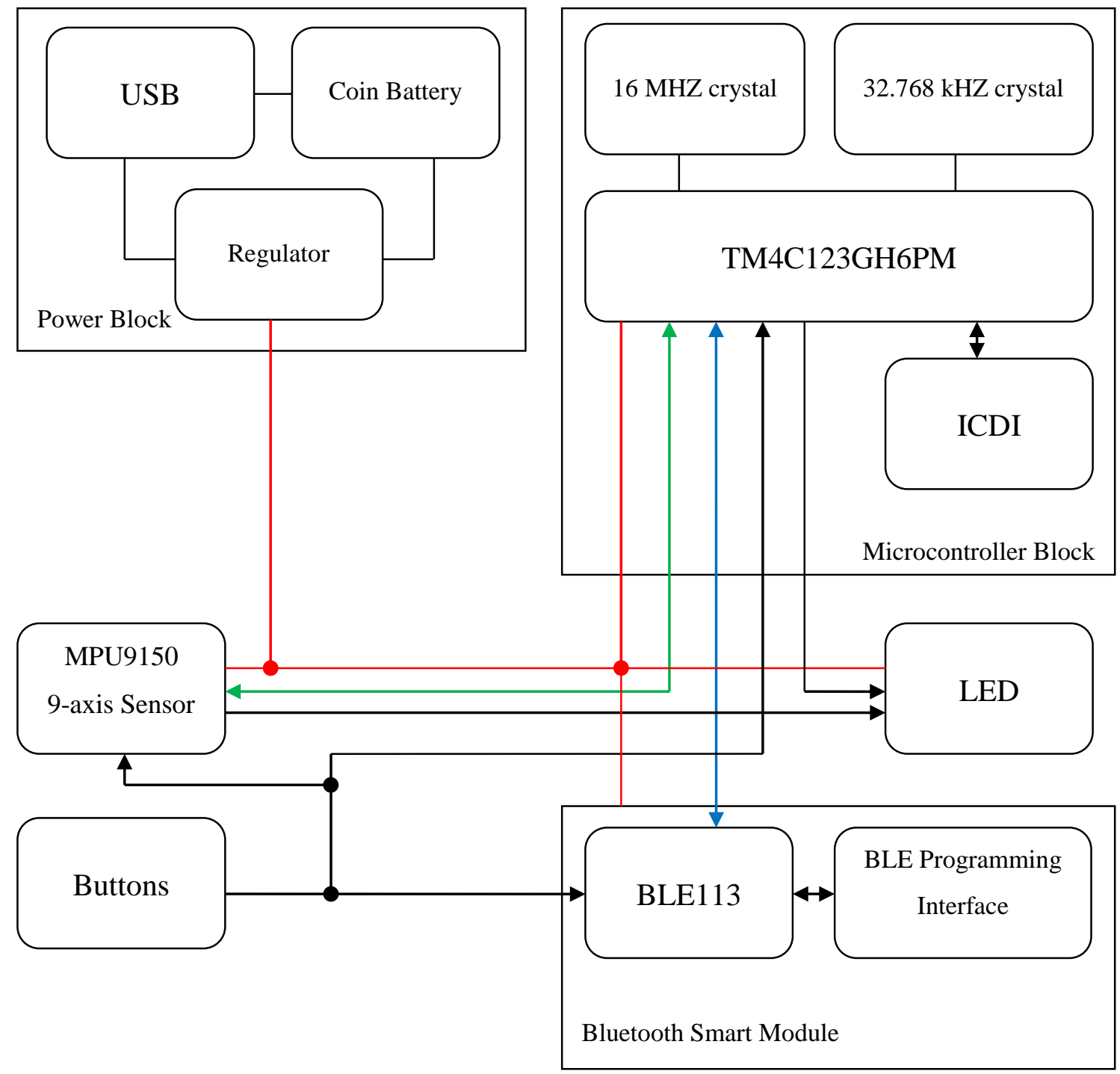

Figure 3: The block diagram of a sensor node in our design. The red line represents 3.3V power lines; the green line represents $\mathrm{I2C}$ channel; the blue line represents UART channel. The arrow represents the flow of data and control. 


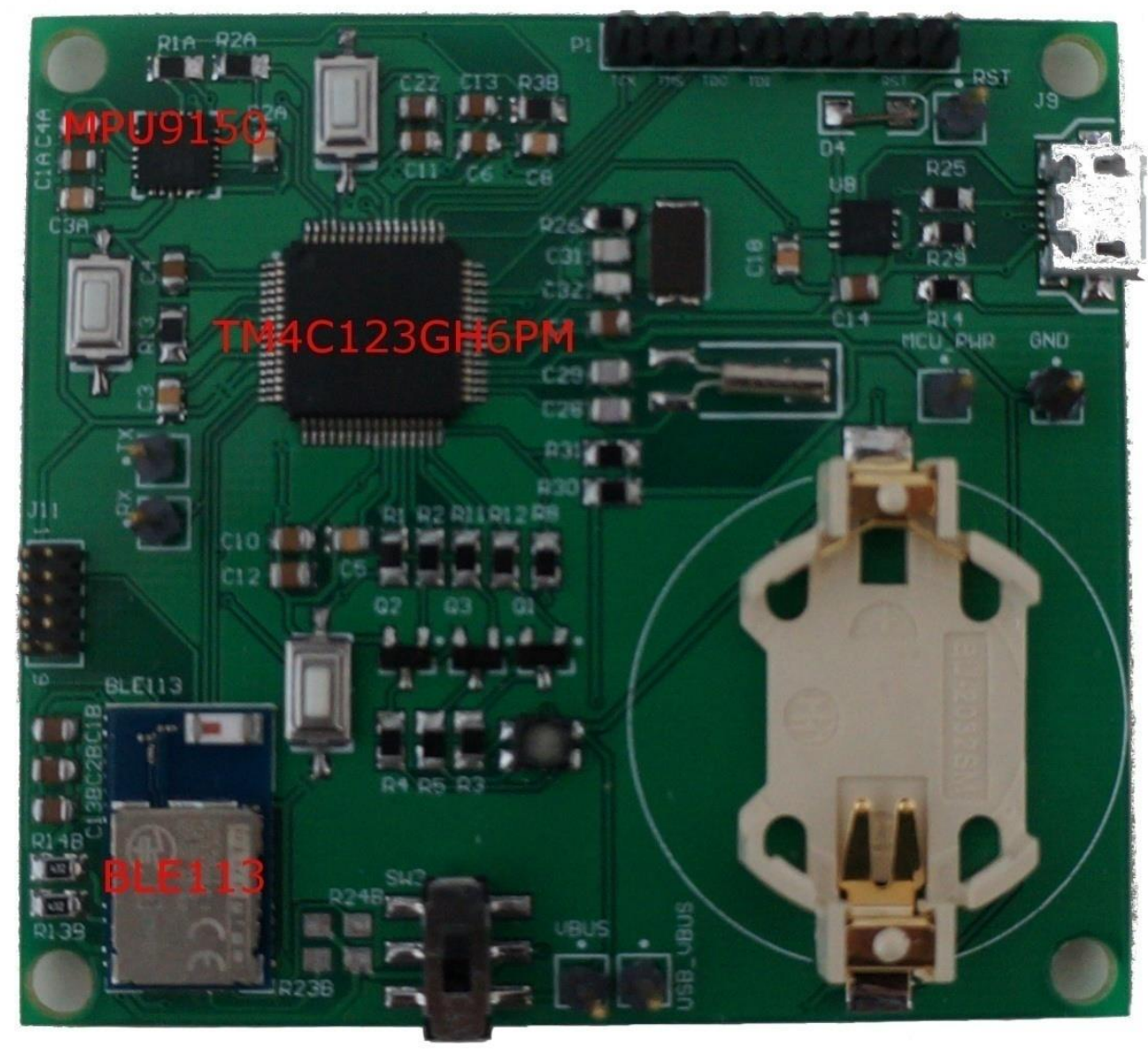

Figure 4: The top view of a sensor node board

\subsubsection{Power}

The sensor node is designed to be powered by micro-USB cable or a rechargeable coin battery. The 5V USB power input is passed into a regulator to output at precisely 3.3V for the microcontroller, LED, the BLE module, and the MEMS motion sensor. The $3.3 \mathrm{~V}$ coin battery output is connected after the regulator with the $3.3 \mathrm{~V}$ line. If both the battery and the micro-USB cable are connected, the battery can be recharged by the USB power input.

A reset button controls the $3.3 \mathrm{~V}$ power input to all these major components. Once the button is pressed the $3.3 \mathrm{~V}$ power line is grounded and all the components are turned off. When the button is released the $3.3 \mathrm{~V}$ power line recovered and all the components are reset. 
The theoretical power consumption of the major components of a sensor node board is listed here in Table 2.

Table 2: Current consumption of major components in working and sleeping modes

\begin{tabular}{|l|l|l|}
\hline & Working & Sleep mode \\
\hline TM4C123G & $\begin{array}{l}\text { Average } 32 \mathrm{~mA} \text { at } 40 \mathrm{MHz} \\
\text { and } 25^{\circ} \mathrm{C}\end{array}$ & $\begin{array}{l}1.4 \mu \mathrm{A} \text { with Real-Time } \\
\text { Clock enabled }\end{array}$ \\
\hline MPU9150 & $4.2 \mathrm{~mA}$ with all sensors on & $6 \mu \mathrm{A}$ when idling \\
\hline BLE113 & $\begin{array}{l}26.1 \mathrm{~mA} \text { at max transmit } \\
\text { power }-23 \mathrm{dBm}\end{array}$ & $0.5 \mu \mathrm{A}$ \\
\hline Total & $62.3 \mathrm{~mA}$ & $7.9 \mu \mathrm{A}$ \\
\hline
\end{tabular}

As shown in Table 2, the total power consumption of the sensor node board is around $62.3 \mathrm{~mA}$ when it is functioning, and only $7.9 \mu \mathrm{A}$ when it is sleeping. It is possible for the sensor node to run on battery for hours continuously or stay in sleep mode for months without charging. Power consumption can be further reduced by lowering the transmit power of BLE113's radio. At $0 \mathrm{dBm}$, the power consumption of BLE113 is only 18.2 $\mathrm{mA}$. There are 3 power levels in total. The signal strength affects the range of the wireless transmission and could be adjusted based on needs. Additionally, the slow-clock mode can be turned on to save more power.

\subsubsection{Clocking}

The sensor node contains two external crystals: a $16 \mathrm{MHz}$ crystal and a $32.768 \mathrm{kHz}$ crystal. These crystals mainly serve the microcontroller. The $16 \mathrm{MHz}$ crystal is part of the main internal clock circuit of the microcontroller, while the $32.768 \mathrm{kHz}$ crystal is for the hibernation feature of the microcontroller. The $16 \mathrm{MHz}$ crystal is adjusted by an internal PLL, which can be configured in software, to achieve higher frequencies for core and peripheral timing. 
The BLE113 module has embedded $32 \mathrm{MHz}$ and $32.768 \mathrm{kHz}$ crystals for independent clock generation.

The MPU9150 sensor also has on-chip timing generator, which has $1 \%$ accuracy for full-temperature range. During testing, the MPU9150's sampling frequency has shown less than $0.1 \%$ of error.

\subsubsection{Programming interface}

The TM4C microcontroller uses an In-Circuit Debug Interface (ICDI) for debugging and programming. The interface can be used to load programs, set breakpoints for debugging, and let the microcontroller print messages to screen.

The BLE113 module has a separate programming interface for configuring the module and the General Attribute Profile (GATT).

The source codes and instructions to program the TM4C microcontroller and the BLE113 module can be found in Appendix B.

\subsubsection{Unit cost}

Cost is one of the most important factors that are considered for this system. The total component cost of a sensor node is $\$ 70.92$ with costs of all components listed in Appendix A. The unit price for the 3 major components are listed below in Table 3; they make up the majority of the total costs. The relatively low cost of the sensor nodes improves the inertial sensor network's potential for consumer-oriented applications. Further cost reduction is possible by replacing the Bluetooth Smart module with a single BLE chip like CC2540, and installing custom antenna.

Table 3: Unit price of major components of the latest purchase from Digikey, before tax, in Canadian Dollar

\begin{tabular}{|l|l|}
\hline Component Name & Component Cost \\
\hline TM4C123GH6PM & $\$ 14.02$ \\
\hline
\end{tabular}




\begin{tabular}{|l|c|}
\hline MPU9150 & $\$ 16.79$ \\
\hline BLE113 & $\$ 21.76$ \\
\hline Total component costs for a sensor node & $\$ 70.92^{1}$ \\
\hline
\end{tabular}

\subsubsection{Physical size}

The sensor node needs to be small enough to be placed on human body without causing too much inconvenience. The prototype board is measured to be $61.9 \mathrm{~mm}$ long and $56.6 \mathrm{~mm}$ wide. This size is a bit larger than desired but it could be significantly reduced by removing power-related section and test points from the prototype board. The battery, the power button, and the regulator could be placed in a separate package so it doesn't affect the sensor parts. Further size reduction could be achieved by placing the components on both sides of the board.

\subsection{Hardware implementation}

Each sensor node is composed of more than 30 different electrical components. The schematics and PCB design can be found in Appendix D. There are 3 main components: TM4C123GH6PM microcontroller, BLE113 module, and MPU9150 9-axis motion sensor. In this section the choice of the components, the specifications, and the actual implementation for each of them are explained.

\subsubsection{Microcontroller: TM4C123GH6PM}

The Texas Instruments' TM4C microcontroller has a powerful 32-bit Cortex-M4 core. Table 4 shows the selected specifications of this microcontroller [17].

\section{Table 4: Selected specifications for TM4C123GH6PM}

\begin{tabular}{|l|l|}
\hline Feature & Description \\
\hline
\end{tabular}

\footnotetext{
${ }^{1}$ The full list of component costs is in Appendix A
} 


\begin{tabular}{|c|c|}
\hline Core & 32-bit Coretext-M4 \\
\hline Performance & 80-MHz operation; 100 DMIPS performance \\
\hline Flash & $256 \mathrm{~KB}$ single-cycle Flash memory \\
\hline System SRAM & $32 \mathrm{~KB}$ single-cycle SRAM \\
\hline $\begin{array}{l}\text { Electrically Erasable Programmable } \\
\text { Read-Only Memory (EEPROM) }\end{array}$ & 2KB of EEPROM \\
\hline $\begin{array}{l}\text { Universal Asynchronous } \\
\text { Receivers/Transmitter (UART) }\end{array}$ & 8 \\
\hline Synchronous Serial Interface (SSI) & 4 \\
\hline Inter-Integrated Circuit (I2C) & 4, allows high-speed mode \\
\hline Controller Area Network (CAN) & 2 CAN $2.0 \mathrm{~A} / \mathrm{B}$ controllers \\
\hline Universal Serial Bus (USB) & USB 2.0 OTG/Host/Device \\
\hline Hibernation Module & Can enter hibernation mode \\
\hline General-Purpose Input/Output (GPIO) & 6 GPIO blocks \\
\hline Analog-to-Digital Converter (ADC) & $\begin{array}{l}2 \text { 12-bit ADC modules, each with a maximum } \\
\text { sample rate of one million samples/second }\end{array}$ \\
\hline
\end{tabular}

TM4C123GH6PM is mainly chosen for its powerful floating-point calculation capability, which is desirable for processing large amount of sensor results real-time, before transmitting the results wirelessly. The FPU supports all kinds of operations: add, multiply, subtract, divide, multiply and accumulate, and square root. 32-bit instructions are provided for single precision (C float, 32 bits) floating-point operations. It also has hardware support for conversion between fixed-point and floating-point data. There are 
dedicated registers reserved for floating-point calculations, storing up to 32 float numbers or 16 double numbers.

The microcontroller also has Nested Vectored Interrupt Controller (NVIC), which can handle large amount of interrupts efficiently with minimal overheads. The NVIC is able to queue the interrupts in order, without risks for losing consecutive interrupts. It is also possible to assign 8 levels of priority to up to 7 system exceptions and 78 interrupts in software. This feature allows the interrupt-based sensors to report readings at high frequency.

Moreover, TM4C123GH6PM has many I/O ports, allowing it to interface with multiple sensors. The 12-bit ADCs outperform most microcontrollers, making the input from analog sensors more accurate. The inertial sensor network may have low duty cycle; therefore the hibernation mode can help conserve battery life if the microcontroller runs on battery.

The microcontroller uses a special debugging interface called ICDI. This interface allows the microcontroller to be debugged by another same microcontroller. In fact, for this project, a TM4C123G LaunchPad is used as the debugger. The microcontroller could also print messages to PC through ICDI. The extensive breakpoint and trace capabilities make developments much easier on this microcontroller.

\subsubsection{Motion sensor: MPU9150}

The InvenSense MPU9150 MEMS sensor is a low-cost 9-axis MEMS sensor which includes a 3-axis accelerometer, a 3-axis gyroscope, and a 3-axis magnetometer. It fits the needs of this project by providing reasonable accuracy, range selections, digital output. In the following table the specification of MPU9150 is compared with a more sophisticated sensor system MicroStrain 3DM-GX3, which costs around \$2,500.

Table 5: Comparison of sensor specifications between MPU9150 and 3DM-GX3

\begin{tabular}{|l|l|l|}
\hline Item & MPU9150 & 3DM-GX3 \\
\hline
\end{tabular}




\begin{tabular}{|c|c|c|}
\hline \multicolumn{3}{|c|}{ Accelerometer Specification } \\
\hline Measurement Ranges & $\pm 2 \mathrm{~g}, \pm 4 \mathrm{~g}, \pm 8 \mathrm{~g}, \pm 16 \mathrm{~g}$ & $\pm 1.7 \mathrm{~g}, \pm 5 \mathrm{~g}, \pm 16 \mathrm{~g}, \pm 50 \mathrm{~g}$ \\
\hline Non-linearity & $0.5 \%$ & $0.1 \%$ \\
\hline Zero Bias & $\begin{array}{l} \pm 80 \mathrm{mg} \text { for } \mathrm{x} \text { and } \mathrm{y}, \pm 150 \\
\mathrm{mg} \text { for } \mathrm{z}\end{array}$ & $\pm 0.04 \mathrm{mg}$ \\
\hline Noise Density & $300 \mu \mathrm{g} / \sqrt{\mathrm{Hz}}$ & $80 \mu \mathrm{g} / \sqrt{\mathrm{Hz}}$ \\
\hline Sampling Rate & $1000 \mathrm{~Hz}$ & $30 \mathrm{kHz}$ \\
\hline \multicolumn{3}{|c|}{ Gyroscope Specification } \\
\hline Measurement Ranges & $\begin{array}{l} \pm 250^{\circ} / \mathrm{s}, \pm 500^{\circ} / \mathrm{s} \\
\pm 1000^{\circ} / \mathrm{s}, \pm 2000^{\circ} / \mathrm{s}\end{array}$ & $\begin{array}{l} \pm 50^{\circ} / \mathrm{s}, \pm 300^{\circ} / \mathrm{s}, \pm 600^{\circ} / \mathrm{s}, \\
\pm 1200^{\circ} / \mathrm{s}\end{array}$ \\
\hline Non-linearity & $0.2 \%$ & $0.03 \%$ \\
\hline Zero Bias & $\pm 20^{\circ} / \mathrm{s}$ & $\pm 0.25^{\circ} / \mathrm{s}$ \\
\hline Noise Density & $0.005^{\circ} / \mathrm{s} / \sqrt{\mathrm{Hz}}$ & $0.03 \% / \mathrm{s} / \sqrt{\mathrm{Hz}}$ \\
\hline Sampling Rate & $8000 \mathrm{~Hz}$ & $30 \mathrm{kHz}$ \\
\hline \multicolumn{3}{|c|}{ Magnetomter Specification } \\
\hline Measurement Ranges & \pm 1200 & $\begin{array}{l} \pm 50^{\circ} / \mathrm{s}, \pm 300^{\circ} / \mathrm{s}, \pm 600^{\circ} / \mathrm{s}, \\
\pm 1200^{\circ} / \mathrm{s}\end{array}$ \\
\hline Sampling Rate & $111 \mathrm{~Hz}$ & $7.5 \mathrm{kHz}$ \\
\hline
\end{tabular}

Table 5 shows that the MPU9150 sacrifice sensor accuracy and sampling rate for lower price. Despite majority of specifications being worse, it is notable that MPU9150's 
gyroscope has lower noise density than 3DM-GX3. The following are a few other comparisons.

- MPU9150 uses ADCs to digitalize the analog outputs: three 16-bit ADCs for gyroscope readings, three 16-bit ADCs for accelerometer readings, three 13bit ADCs for magnetometer readings. 3DM-GX3 also uses 16-bit ADC.

- MPU9150 has a very robust 10,000 g shock tolerance, while 3DM-GX3 only has $500 \mathrm{~g}$ shock tolerance

MPU9150 could also help save power for the sensor node as well, because of its 1024 B FIFO buffer which allows data to be read by the system processor in bursts. Then the system processor could enter sleep mode while the MPU9150 continues to collect data to fill the buffer.

\subsubsection{Bluetooth Smart module: BLE113}

The Bluegiga BLE113 module is based on Texas Instruments' CC2541 chip, and has all the features required for a Bluetooth Smart application. The following components are integrated in the module: a Bluetooth Smart radio, software stack, and GAP, GATT support. BLE113 is compliant to wireless certifications of all major countries in the world. The radio has transmission power from $0 \mathrm{~dB}$ to $-23 \mathrm{~dB}$, and receiver sensitivity of $-93 \mathrm{~dB}$. The module consumes ultra-low current, as low as $500 \mathrm{nA}$ while in sleeping mode, and can wake up in less than a couple of milliseconds. During transmission, the module's current consumption is between $14.3 \mathrm{~mA}$ and $27 \mathrm{~mA}$. The module uses UART as the primary interface with an external processor, and it also has peripheral interfaces of SPI, I2C, PWM, and GPIO. The module even has a 12-bit ADC. These peripheral interfaces and the ADC are for the microprocessor incorporated inside the module.

BLE113 contains a Texas Instruments' 8051 microprocessor core which could be used for standalone operations. It has 8 KB SRAM, and 128/256 KB Flash memory. However, this microprocessor core could only be programmed with BGScript, a special script for Bluegiga modules, which is unable to handle complicate programs and configurations. Furthermore, there is no debugger for this microprocessor, making the 
development process much less convenient. These problems significantly limit the 8051 core's already relatively poor performance. Hence this integrated microprocessor is not utilized for this project.

\subsection{Microcontroller software Design}

The sensor node contains a powerful Cortex-M4 microprocessor that supports software with many floating point manipulations. The software is primarily developed to capture sensor measurements and send them to the BLE module for transmission. Additional features such as basic data processing and sensor fusion could be added to reduce the bandwidth needed for transmission without compromising data accuracy and timeliness.

In this section, the basic operations in the software that are needed for the sensor node to work is presented.

\subsubsection{Initialization and configuration}

The microcontroller software program is developed in Texas Instruments' Code Composer Studio v5.2 environment, with library for TM4C123GH microprocessor.

At start, the software is programmed to initialize the pointers to store the sensor readings and sensor fusion results. There are at most 16 float data coming out from the MPU9150 sensor for each iteration. Among these 16 floats, 3 floats are for 3-axis accelerometer data, 3 floats are for 3-axis gyroscope data, 3 floats are for 3-axis magnetometer data, 3 floats are for the Euler angle outputs, and the last 4 floats are for the Quaternion results. This arrangement allows all sensor data and sensor fusion results to be collected and calculated.

Next the microcontroller system properties are configured and the I/O ports with MPU9150 and BLE113 are initialized, as shown in Figure 5. The system clock is set to $40 \mathrm{MHz}$ from PLL with $16 \mathrm{MHz}$ crystal reference. GPIO A is set up for UART0, which is used to print messages to PC via ICDI for debugging. Port A0 is configured to be the receiving pin and port $\mathrm{A} 1$ is configured to be the transmitting pin. The $16 \mathrm{MHz}$ oscillator 
is used as the clock source for this UART channel, and the baud rate is set to be 115,200. UART4 channel for communication with the BLE113 module is set up in similar way, with port $\mathrm{C} 4$ as the receiving pin and port $\mathrm{C} 5$ as the transmitting pin. UART4 does not use flow control, but instead transmits data in packet mode. This is to simplify the communication with BLE113 module. The baud rate of UART4 is also 115,200, same as UART0. To trigger the corresponding method whenever a new event or response comes from the BLE113 module, the UART4 is set to interrupt the main program once a receive event or a receive timeout event happens. 


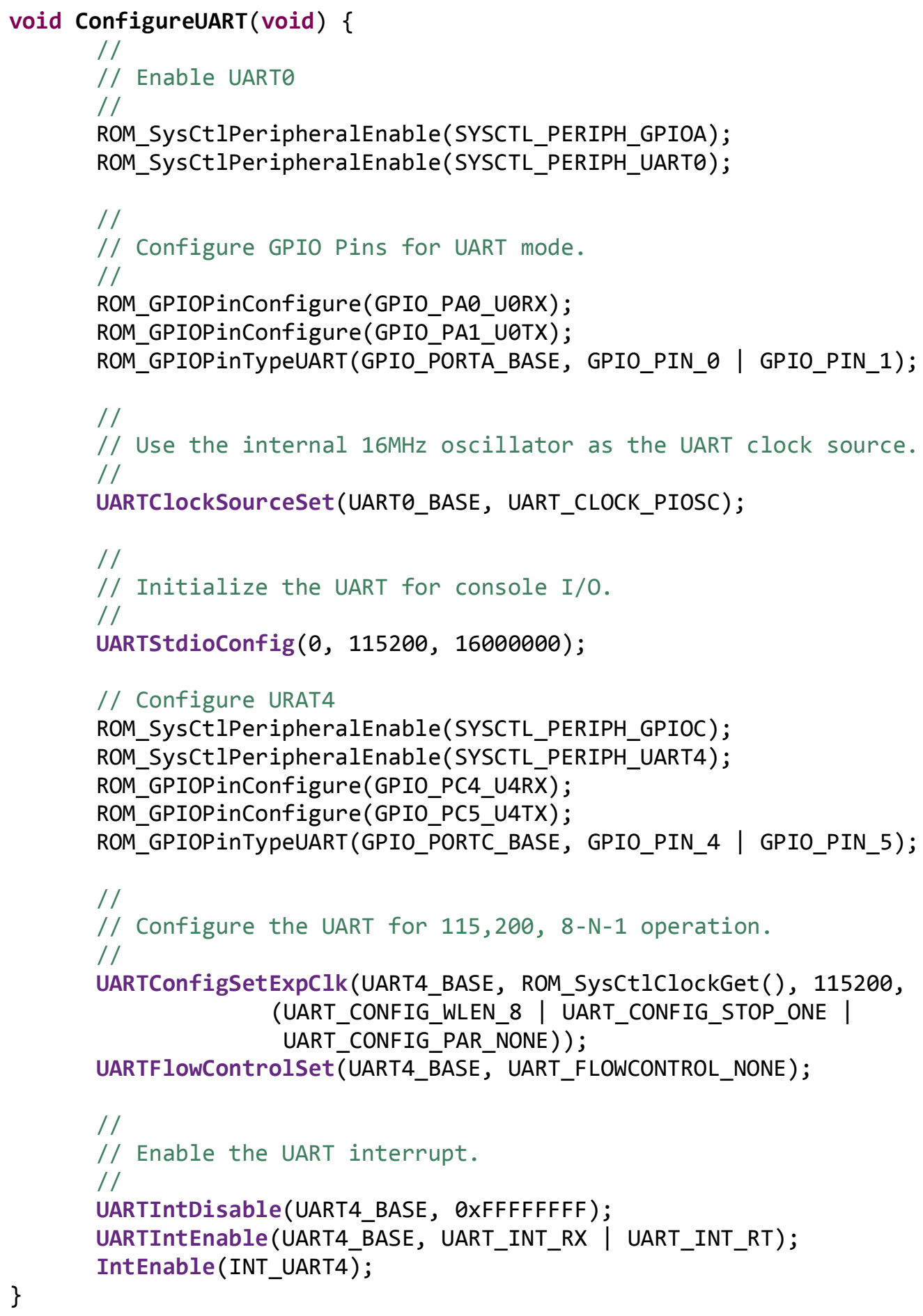

Figure 5: Code snippet for configuring UART channels 
After the UART4 channel is set up, a few library functions are called to initialize BLE113 module for connection with a remote BLE device. A few global variables, g_bleUserFlag, g_bleFlag, and g_bleDisconnectFlag, form the state machine for BLE113 module. Before initialization, all flags are set to 0 (false). The initialization starts by calling ble_cmd_system_reset method, which sends command to reset the BLE113 module. Once the reset is complete, the response comes back, generating a system boot event. Then the program continues to set GAP mode and bondable mode in sequence, as shown in Figure 6. If the response comes back indicating the commands fail, then the program resets the BLE113 module again and repeats the process. When both GAP mode and bondable mode are set successfully, the BLE113 becomes ready for connection with remote BLE device. At this time, g_bleFlag is set to 1 (true) so the program knows the BLE113 module is ready. The details about sending commands and interpreting responses are described in sub-section 3.3.3 of this chapter.

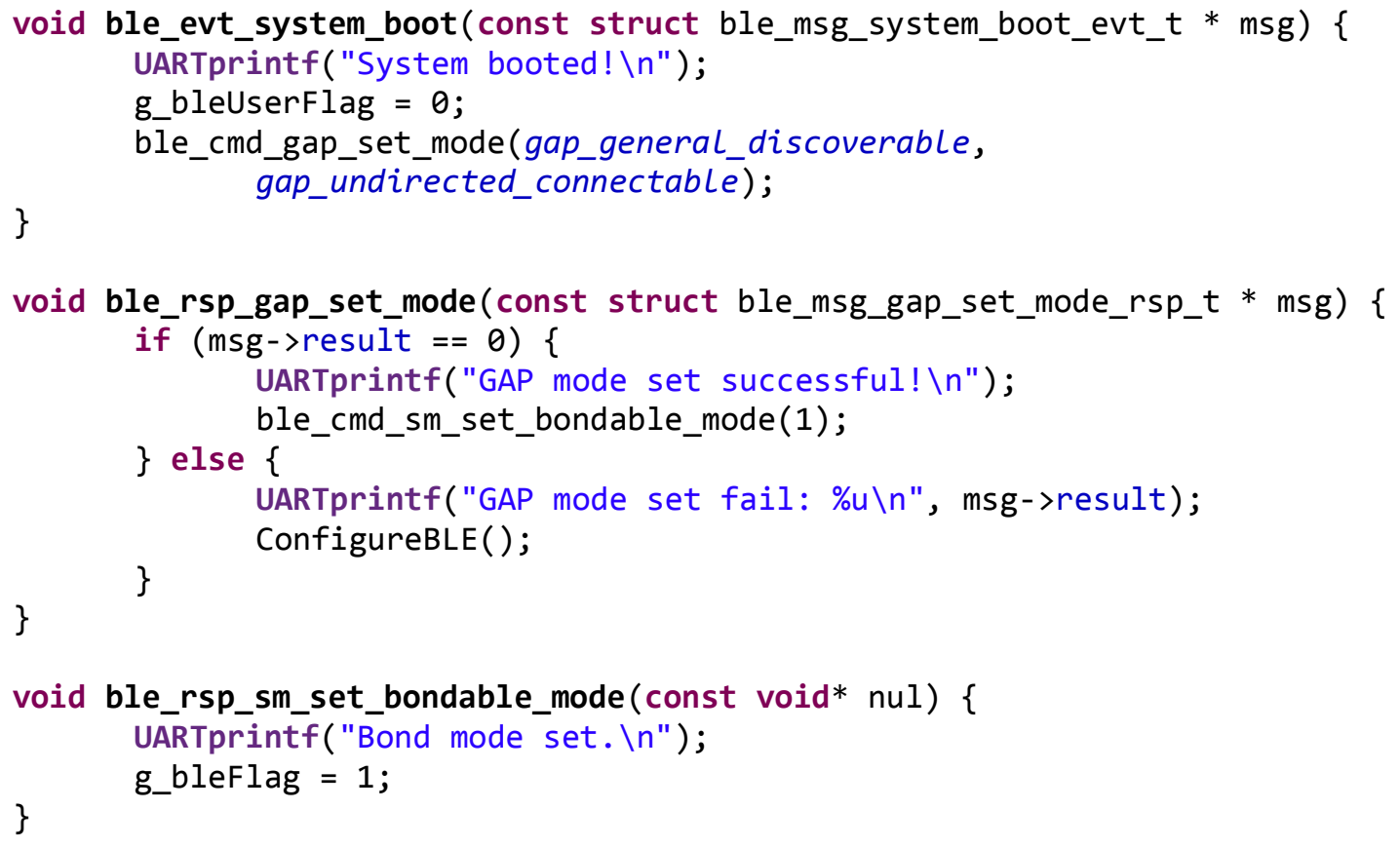

Figure 6: Code Snippet for preparing the BLE113 module for connection 


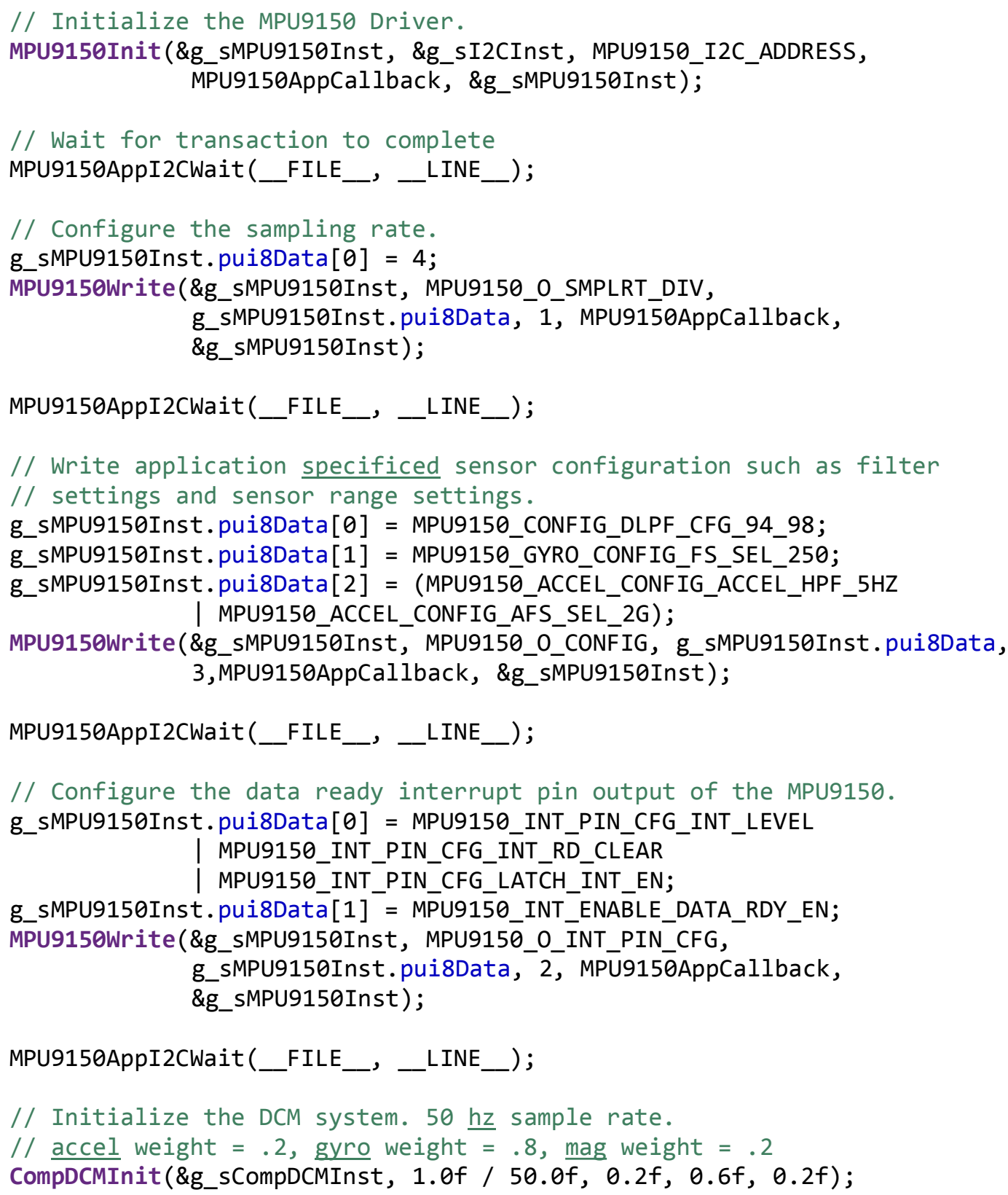

Figure 7: Code Snippet for the MPU9150 initialization sequence 
Since the UART channels and BLE113 modules are initialized and ready, next step is to implement the initializations of I2C channel and MPU9150 sensor. The microcontroller communicates with MPU9150 through I2C3 channel residing on GPIOD port. The SCL pin is set to be D0 and the SDA pin is set to be D1. GPIOE port E2 pin is used for interrupts from MPU9150, with the interrupt mask set to falling edge only. A system function ROM_IntMasterEnable is called to enable all interrupts to the microprocessor. The $\mathrm{I} 2 \mathrm{C} 3$ peripheral is then initialized, allowing commands to be sent from the microcontroller to MPU9150, as illustrates in Figure 7. At first, the initialization command is sent to MPU9150, with pointers of driver instances and callback. For each command, the program needs to wait for the response before sending a new command. If the wait times out, the program reports the error by setting the LED to blink red light with $10 \mathrm{~Hz}$ frequency. The second command is to set the sampling rate. The parameter integer plus one is used to divide $1 \mathrm{KHz}$. For example, the result sampling rate would be $200 \mathrm{~Hz}$ if the parameter is 4 . The third command sets specific sensor configurations such as range selections. It also sets the filters for both accelerometer and gyroscope. The forth command configures the data ready pin output for MPU9150 so it can successfully generate an interrupt to the microprocessor. The last command initializes the Direction Cosine Matrix (DCM) calculation, allowing the program to set sampling frequency and specify weights for accelerometer, gyroscope, and magnetometer. The DCM library is included in the example code provided by Texas Instruments as part of the software stack for the TM4C microcontroller. The algorithm in DCM library is basically a complementary filter that can calculate Euler angles and Quaternions from the outputs of a 9-axis sensor. There's a global flag indicating whether the DCM algorithm is running.

In the last section of the initialization, the tri-color LED is configured to blink at 1 $\mathrm{Hz}$, with half the intensity and white color. Then the EEPROM is initialized so that the program can read the configurations saved in EEPROM. The EEPROM could also save the error estimations calculated by the calibration process for the sensor node.

With the initializations done, the program enters an infinite loop, continuously reading data from MPU9150 and waiting for connection from the remote data processing device. 


\subsubsection{Data and control flow}

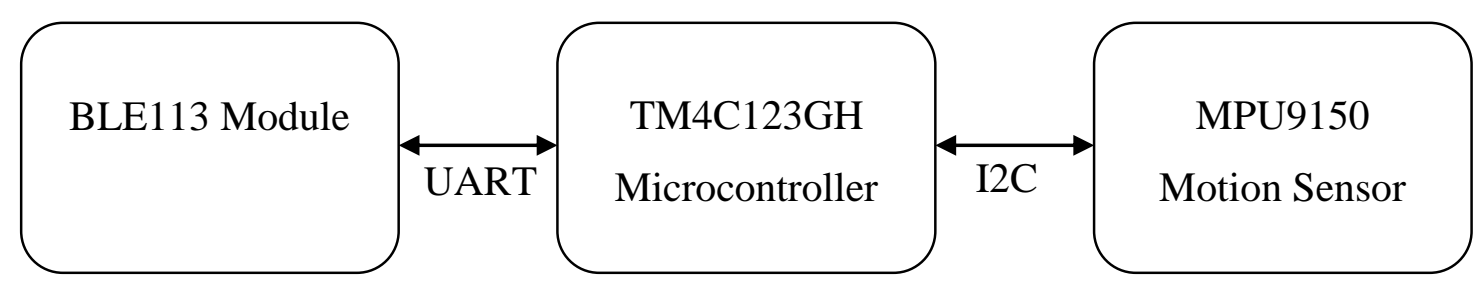

Figure 8: The figure above shows the data flow from sensor to the Bluetooth module.

Figure 8 shows communication protocol between the TM4C123GH microcontroller and the BLE113 module, MPU9150 sensor. After initialization, the system is designed to continuously read the measurements from the sensor and transmit the data to remote data processing device if it's connected.

Every iteration of the loop, the software is programmed to loop check a flag for the sensor data to arrive. Whenever a new measurement is ready to read, the interrupt pin E2 is forced to low voltage by MPU9150 to alert the microcontroller. Detecting the falling edge, the interrupt service routine is invoked and the flag is set to true to break the loop. Then the system sends a read request to accelerometer, gyroscope, magnetometer each, and the measurements are transmitted to the microcontroller over $\mathrm{I} 2 \mathrm{C}$. This mechanism is there to ensure the data are read at desired frequency.

At this point, the program already has the raw data from the 9 -axis sensor. The microcontroller could do some basic processing, such as removing biases in sensor measurements, before sending the data. It is also possible to apply very complicate filters to the results for better accuracy. In addition the microcontroller can calculate Euler angle and Quaternion data with the DCM library. The DCM algorithm passes the data from accelerometer, gyroscope, and magnetometer into a complimentary filter. The result is a rotation matrix, and further calculation could yield Euler angle and Quaternion results. The main purpose of doing some data processing at sensor node is to reduce the amount of data being transmitted to the remote BLE device. If applications only require Euler angles or Quaternions, there is no need to transmit raw sensor readings. 
For transmission of the data, if the Bluetooth Smart module has already indicated that the remote data processing device is connected, the global flag g_bleUserFlag is set to 1 (true). Then the data can be transmitted to BLE113 via UART and written to the corresponding attribute in the GATT table. For each type of sensor or DCM results, the program needs to send one attribute write command. If the system requires transmitting more than one type of results, then in the same cycle the next transmission must wait until the response to previous transmission is received. Otherwise, the UART channel between the microcontroller and the BLE113 module might be jammed with excess data, causing problems in future transmissions. The reason is, when there are too much data in transmission, the BLE113 module might miss one byte or more, causing the module to wait on the missing bytes even after the transmission is finished.

If the connection is broken due to operations on the remote device or going out of range, the microcontroller is notified of the event by the BLE module, setting the global flag g_bleUserFlag to 0 and $g \_$bleDisconnectFlag to 1 . By this way in next iteration the program will detect the flags, stop attempting to transmit the data and call the method to configure the BLE113 module for reconnection. Therefore, even if a disconnection happens (most likely due to sensor node moving out of range), a new connection can be established as soon as the pair of devices are ready and in range.

\subsubsection{Implementation of BGLib}

The BGLib is the C library for the Bluegiga BLE113 to work with external microprocessor. It contains various commands, events, responses, and their corresponding UART channel messages. When sending a command, the library functions translate the command to a message and send it over UART; when receiving an event or response, the library functions interpret the message received and trigger the correct method to handle the received event or response. The implementation of BGLib requires writing two methods: one for input from and one for output to the UART channel.

The input method is designed to receive data from the UART channel, interpret the header of the message, and call the handler from BGLib to process the message. Since the message coming from BLE113 does not have fixed length, the program needs to 
figure out how long the message is in total. Whenever an interrupt happens for UART4, the input method is called. The first 4 bytes of the message are read and saved into a header struct. The header struct determines the type of the message and how many bytes of payload the message has. The BGLib functions are called to transform the header struct to a constant struct type called ble_msg, which points to the corresponding handler function according to the type of the message. Then the program could read an exact number of bytes as the payload, and save them in a separate data array. Finally the handler function is invoked with the payload data array as a parameter. This handler function is declared in BGLib and implemented in the main program. Since the message is from BLE113 to the microcontroller, it would be either an event or a response. If the message is an event, then the program could do something in response to it; if the message is a response for a previously sent command, then the program could find out whether the command was executed successfully or not. Figure 9 shows the implementation of some of the handling methods in the main program. The events such as boot-up, connection, and disconnection would trigger the program to initialize BLE113, set the flag to allow data transmission to it, and prepare it for reconnection respectively. The response ble_rsp_gap_set_mode leads to sending next command in initialization sequence, ble_cmd_sm_set_bondable_mode to BLE113. Another response ble_rsp_attributes_write leads to setting the flag indicating new write command could be sent to BLE113 now. 


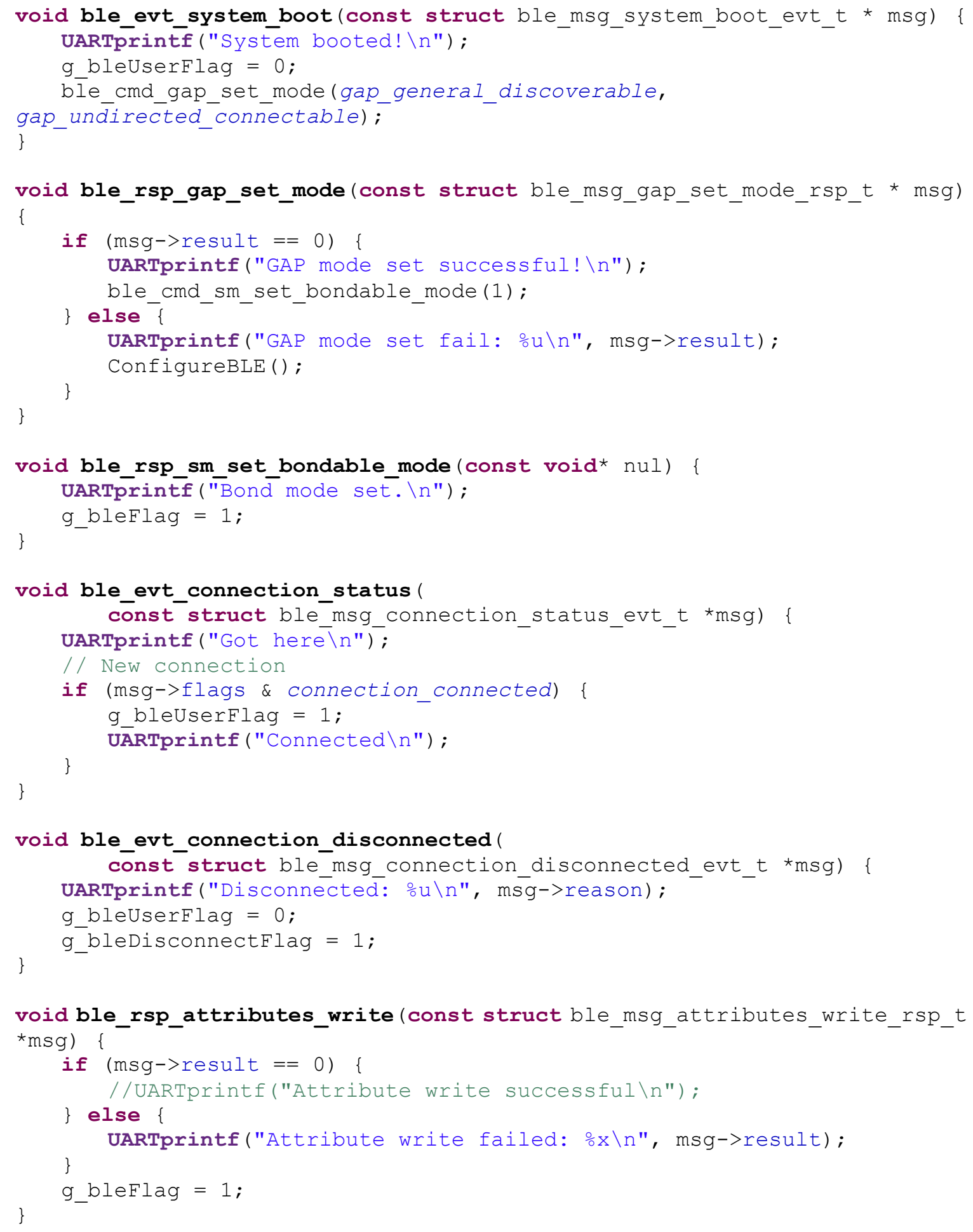

Figure 9: Code snippet for some of the handling methods of BLE events and responses 
For the output method, its responsibility is to output messages to the UART channel in the correct format. By design, the output method is not directly called by the main program. For every specific command, a BGLib method is defined to arrange the message for the command, and sends the message to the abstract method bglib_output. When the main program wants to send a command to BLE113, the corresponding BGlib method is called. To connect the BGLib function with the user-defined output method, the pointer of bglib_output is assigned to the pointer of ouput during initialization. This way when the BGLib calls the bglib_output function, it is actually the output function that gets called. As stated in sub-section 3.3.1, the UART communication from the microcontroller to BLE113 is in packet mode. This means that for output, before sending the actual message, the total length of the message needs to be sent first. Then, the actual message can be written to the UART4 channel in two parts. The first part is the 4-byte long header, which contains the message type, the number of bytes of payload, and other configurations. The second part is the payload, such as the collected sensor data ready to send to the remote data processing device. The nature of this type of communication requires time separation between transmissions. Normally it's only safe to transmit a new message after the response to the previous message is received.

To finish the implementation, all the relevant events, responses are handled by the system. Table 6 shows the necessary events, responses, commands needed for the program to achieve basic functionalities.

Table 6: Commands, Events, and Responses for sensor node to have basic functions

\begin{tabular}{|l|l|l|}
\hline Name & Type & Function \\
\hline ble_cmd_system_reset & Command & Reset the BLE113 module \\
\hline ble_cmd_gap_set_mode & Command & Configure the GAP mode \\
\hline ble_cmd_sm_set_bondable_mode & Command & Set BLE113 to bondable mode \\
\hline ble_cmd_system_hello & Command & $\begin{array}{l}\text { Expect BLE113 to respond with } \\
\text { ble_rsp_system_hello }\end{array}$ \\
\hline
\end{tabular}




\begin{tabular}{|l|l|l|}
\hline ble_cmd_system_get_info & Command & $\begin{array}{l}\text { Expect BLE113 to respond with } \\
\text { ble_rsp_system_get_info }\end{array}$ \\
\hline ble_cmd_attributes_write & Command & $\begin{array}{l}\text { Send data to the corresponding } \\
\text { GATT attributes }\end{array}$ \\
\hline ble_rsp_gap_set_mode & Response & $\begin{array}{l}\text { Indicate whether GAP mode is set } \\
\text { successfully }\end{array}$ \\
\hline ble_rsp_sm_set_bondable_mode & Response & $\begin{array}{l}\text { Indicate BLE113 has received } \\
\text { ble_cmd_sm_set_bondable_mode }\end{array}$ \\
\hline ble_rsp_system_hello & Response & $\begin{array}{l}\text { Indicate BLE113 has received } \\
\text { ble_cmd_system_hello }\end{array}$ \\
\hline ble_rsp_system_get_info & Response & $\begin{array}{l}\text { Contain information for build } \\
\text { number, protocol version, and } \\
\text { hardware }\end{array}$ \\
\hline ble_event_connection_disconnected & Event & $\begin{array}{l}\text { Indicate connection disconnected, } \\
\text { contain the reason for disconnection }\end{array}$ \\
\hline ble_rsp_attributes_write & Eventevt_connection_status & $\begin{array}{l}\text { Indicate whether the write is } \\
\text { successful }\end{array}$ \\
\hline contain the current connection status
\end{tabular}

\subsubsection{Simple calibration}

The MPU9150 sensors sometimes have significant errors in raw measurements, therefore before actually deploying the system, all the sensor nodes should be individually calibrated to estimate the most common errors: zero biases and scale factors. 
However the basic calibration procedures of the embedded accelerometer, gyroscope, and magnetometer are vastly different. For the accelerometer, the approach is to measure the output of one axis with actual gravity equal to $-\mathrm{g}, 0$, and $\mathrm{g}$. It is then possible to use these measurements to calculate the zero bias and scale factor for each axis. For the gyroscope, the zero biases are basically the stationary readings, while the scale factors require integrating the measurements as the sensor rotates a certain angle around each axis. Calibrating the magnetometer, on the other hand, is much harder. Rigorous magnetometer calibration involves taking thousands of measurements of different orientations in a 3D space.

A simple calibration procedure is developed for the project. It is included in the microcontroller software of every sensor node. The aim is to measure the accelerometer zero biases and scale factor, plus the gyroscope zero biases. The magnetometer calibration is not possible with the sensor node alone, therefore it is omitted. It is also difficult to perform accurate measurements for gyroscope scale factors since it requires integrating the noisy readings. The calibration mode is programmed to start as the calibration button (one of the two programmable buttons) on the sensor node board is pressed. The calibration process has 3 stages, each with different placement of the sensor node. In the first stage, the $\mathrm{z}$ axis points downward along the direction of the gravity. The second and third stage are for the $\mathrm{y}$ and $\mathrm{x}$ axes respectively. The LED changes color to indicate different stages, and the calibration button needs to be pressed to proceed to next stage. Readings from the accelerometer and the gyroscope are averaged for every stage to get measurement values of the accelerometer corresponding to $0\left(a_{0}\right)$ and gravity $\left(a_{g}\right)$ along each axis, and zero biases for the gyroscope. While zero biases of the accelerometer are just $a_{0}$, scale factors for the accelerometer is calculated using the following equation.

$$
\text { scale factor }=\frac{\left(a_{g}-a_{0}\right)}{g}
$$

Once complete, the microcontroller saves the correction values in EEPROM for realtime use with the raw sensor data to produce more accurate estimates. 
However, this simple calibration method could introduce errors because the sensor axes might not be perfectly aligned with the gravity during calibration. Hence the calibration process needs to be carefully executed to minimize the errors.

\subsection{Bluetooth Smart module configuration}

The BLE113 module is programmed separately from the rest of the system by attaching a Texas Instruments' $\mathrm{CC}$ debugger to the $2 \times 5$ pin heads on the sensor node board, and connecting the $\mathrm{CC}$ debugger to PC. Bluegiga provides tools to compile and upload the configuration files to BLE113. There are 3 configuration files: cdc.xml, gatt.xml, and hardware.xml. Several examples of working systems are provided as well. Based on an example for interacting with external microprocessor, only settings in gatt.xml and hardware.xml need to be modified to adapt to our system, and cdc.xml can be left untouched.

\subsubsection{Configurations in hardware.xml}

Figure 10 shows the content of the modified hardware.xml for the sensor node.

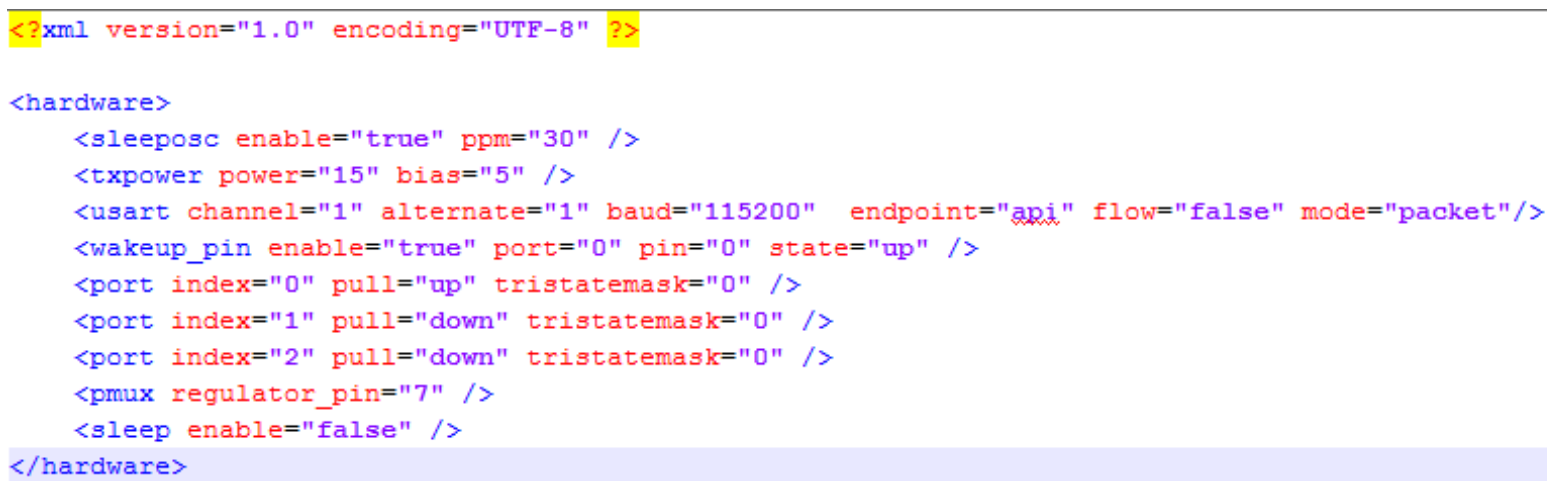

Figure 10: Content of hardware.xml for the sensor node

The most important part of this document is the UART configuration on the third line. This line specifies which UART channel to communicate with the external microprocessor, the baud rate of the channel, the flow control status, and whether the transmission is in packet mode or not. This line also tells the BLE113 module to execute the BGAPI commands by making the endpoint "api". BGAPI can interpret incoming 
UART messages and execute accordingly. BGAPI can also output message to UART channel to report an event or respond to a command. These settings need to be matched with the implementation of the microcontroller software.

Other lines are mostly unchanged from the original example provided by Bluegiga. Note that sleep is disabled for debugging purpose. It can be enabled, but then before every transmission, the microprocessor needs to send a signal to the wakeup pin as specified. In this case if sleep is enabled, the microprocessor needs to send a rising edge signal to port 0 pin 0 of the BLE113 module to wake it up.

\subsubsection{Configurations in gatt.xml}

The gatt.xml determines configurations for the Generic Attributes Profile (GATT). The GATT stores data, also called attributes, in characteristics. A characteristic contains:

- Characteristic Declaration - configurations that describe the properties of the Characteristic Value, its Universally Unique Identifier (UUID) and handle for access

- Characteristic Value - a value with known format

- Characteristic Descriptor - additional text that provides information about the characteristic

The property of characteristic value regulates how the data could be transmitted. There are mainly 5 types of properties: const, read, write, notify, and indicate.

- Const means the characteristic value is constant.

- Read is for the remote device to actively send a read command to the BLE113 module, and gets the current value in response from the module.

- Write is for the remote device to modify the value in this characteristic. 
- Notify, when enabled by remote device, lets the BLE113 module automatically send the data in characteristic to the remote device, whenever new values come in from the microprocessor.

- Indicate is like notify, but the remote device need to send a confirmation each time the BLE113 module sends a value.

- Other properties include authenticated read and write, adding an authentication requirement for the read and write operations.

Several characteristics could be grouped together as a service. Each service and characteristic needs to have a unique randomly-generated UUID for reference.

In our sensor node system, the GATT has a simple structure. The first service provides information about the BLE113 module, such as device name and reconnection address. The second service contains information of the hardware, including the manufacturer, the model name, and the serial number. The third service is for battery information. The next 3 services correspond to each individual sensor: accelerometer, gyroscope, and magnetometer. Each service contains one characteristic that stores 12 bytes long data, enough to transmit 3 float results, one for each axis. It is arranged in this way so that it only takes one write to transmit all the data for one type of sensor results to the BLE113 module, reducing the traffic on the UART channel between the microprocessor and the BLE113 module. Then there is the service for the Euler Angle output from DCM library. Similarly, the Euler angle service has a characteristic of 12 byte length, enough to hold all results. 


\section{Chapter 4: Application Example - Personal Navigation System}

An example application, MotionTracker, is designed to demonstrate the advantages of the inertial sensor network proposed in this thesis. The core concept is to use the Euler angles measured by the sensor nodes placed on a person's legs to determine the angles of legs relative to the ground, and find out the step length using trigonometry. This is similar to human motion tracking. E. R. Bachman et al. designed an efficient motion tracking system for humans and robots, using Quaternions calculated by a complementary filter [18]. The system is able to accurately track the orientation of a rigid body with a large sensor pack that measures $10.7 \mathrm{~cm}, 5 \mathrm{~cm}$, and $3.7 \mathrm{~cm}$ for length, width, and height. It is not practical to wear such a large sensor on the human body. Another research from Tsinghua University uses a Kalman filter to compute the orientation of human body segments [19]. In the experiment they let a subject move arm horizontally and vertically, with sensors placed on the subject's shoulder, upper arm, and lower arm. They were able to achieve an error of less than $1 \mathrm{~cm}$ for all axes when results are combined from 3 sensors. However, the data output of the system has a 0.9 second delay. Compared to these examples, the MotionTracker model is simplified to just account for the walking associated lower body motion. With this constraint, Euler angles can be used without worrying about the singularities. Unlike Quaternions, the Euler angles can be directly used for calculation to find step length. The MotionTracker system is designed to report real-time Euler angle data of the legs with minimum delay through Bluetooth Smart to users' smartphones.

As shown in Figure 11, MotionTracker is composed of a remote data processing device and at least 4 sensor nodes which measure the angles of the limbs relative to the gravity. These 4 sensor nodes are tightly attached to the legs of the user. The legs are divided into 4 parts, the upper left leg (part 1), lower left leg (part 2), upper right leg (part 3), and lower right leg (part 4); each part has one sensor node on the front, placing around the knees, with its y direction facing upward along the leg, its $\mathrm{x}$ direction toward the right side, and its $\mathrm{z}$ direction going into the leg. Each sensor node computes the angle of the part of leg it attached to and transmits the results to a smartphone, via Bluetooth Smart. 
The smartphone is then able to synthesis and analyze the data from all the sensors, and determines if a step is complete. Once a step is complete, with pre-input data about the user's limb length, it is possible to compute the step length.

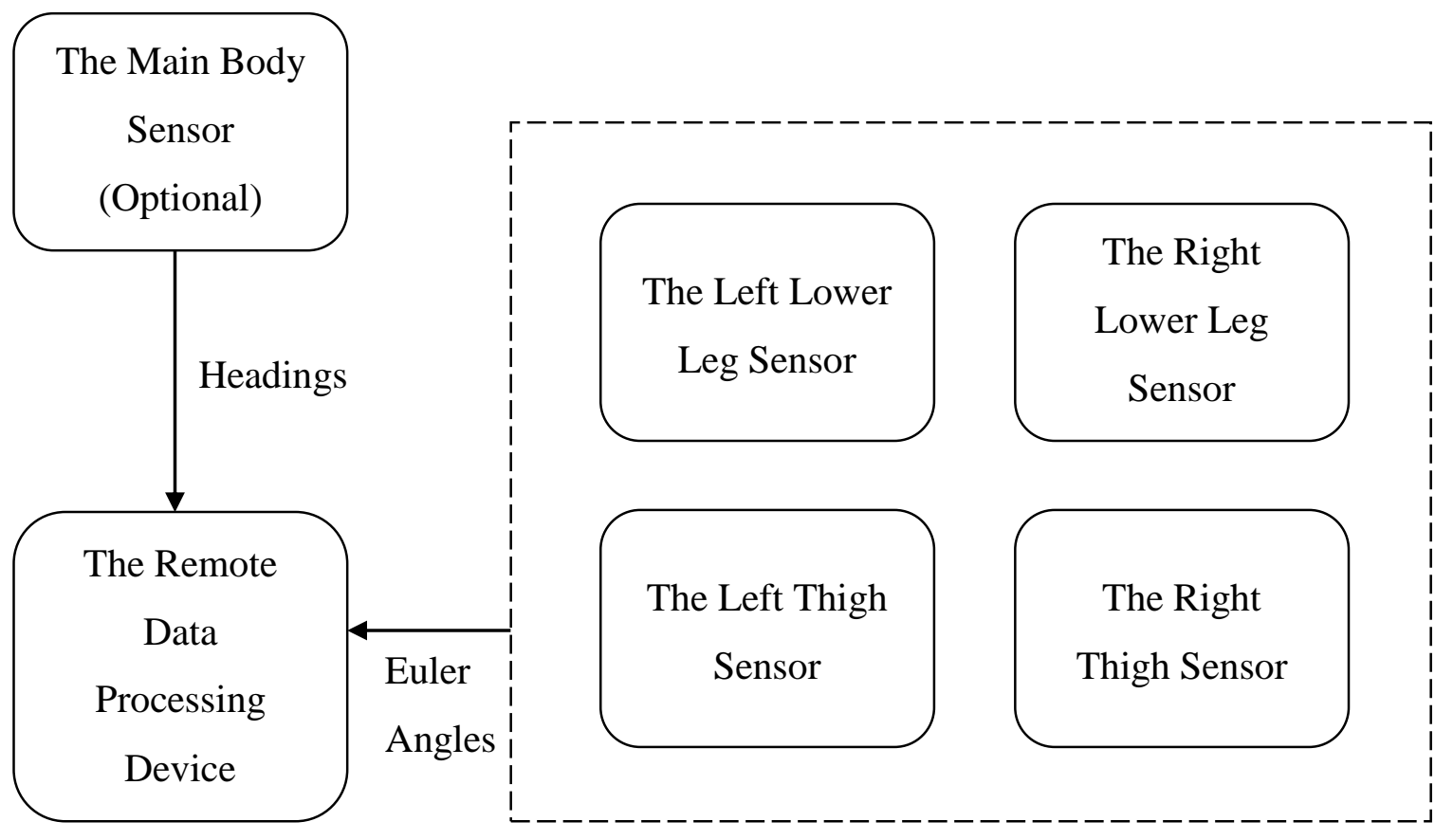

Figure 11: The general architecture of the MotionTracker system. The Main Body Sensor SMB1 provides headings data. Each limb sensor reports its orientation in Euler angles.

In addition to the 4 basic sensor nodes, an additional sensor node can be attached to the waist to measure the heading of the pedestrian. The current heading is applied on the step at the same time a new step is determined. A continuous array of the directed steps can form a path on 2D surface, allowing the system to track the pedestrian as long as the initial position and heading are given. However in the experiment this sensor was not used due to the magnetometer of MPU9150 unable to produce measurements with reasonable accuracy.

Most of this chapter deals with two major problems faced by this example application: 
- How to determine the current step length?

- How to differentiate between steps?

After that, more discussions about the software implementation of remote data processing device are followed.

\subsection{How to determine the current step length}

To measure the step length, the length of legs must be known. As Figure 12 shows, the lengths of the upper legs are denoted as $\mathrm{L}_{1}$ (left), $\mathrm{L}_{3}$ (right), and the lengths of the lower legs are denoted as $\mathrm{L}_{2}, \mathrm{~L}_{4}$. These lengths are measured from joint to joint for best accuracy; for example, $\mathrm{L}_{2}, \mathrm{~L}_{4}$ are measured from ankle to knee.

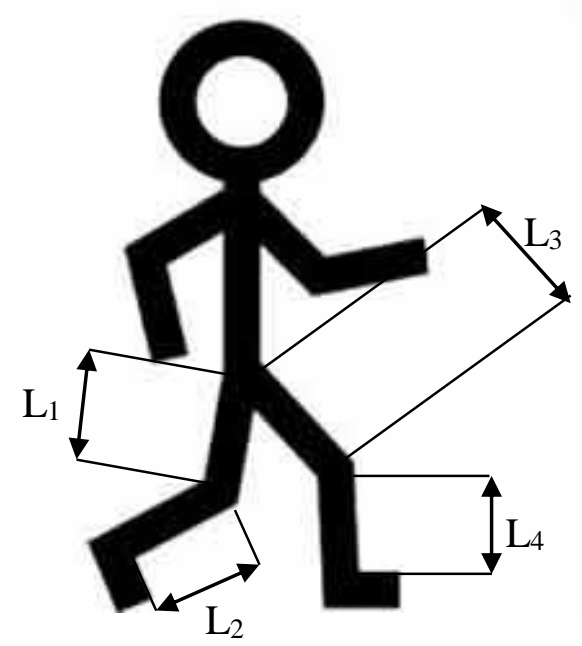

Figure 12: A pedestrian stepping with his right leg

The Euler angles are most commonly used to describe the orientation of an object. In the context of this example application, the Euler angles describe the orientation of the sensor nodes, and the limb they attach to. By definition, the roll of the Euler angles represents the rotation of sensor node board around X'-axis from the ground. The X'-axis is the result of rotating the $\mathrm{X}$-axis of Earth reference frame by the yaw angle (heading). This angle is most useful in the calculation of step length, and it is denoted as $\mathrm{E}_{1 \mathrm{x}}$ for upper left leg. The pitch of the Euler angles represents the relative angle around z-axis of 
the sensor between the sensor node board and the ground. It is as denoted $\mathrm{E}_{1 \mathrm{y}}$ for the same limb. The yaw of the Euler angles represents the heading of the sensor node on the ground plane (around Z-axis of Earth frame). It is denoted as $\mathrm{E}_{1 \mathrm{z}}$. The Euler angles for other parts of the legs are denoted similarly. Based on these results, the system could calculate the horizontal displacements of the two feet $\left(\mathrm{D}_{\mathrm{L}}, \mathrm{D}_{\mathrm{R}}\right)$ along main direction (front and back) relative to a virtual origin. Figure 13 below denotes Euler angles and horizontal displacements of the two feet along the primary direction. Using trigonometry, the displacements can be calculated as follows:

$$
\begin{aligned}
& D_{L}=\cos E_{1 x} \times L_{1}+\cos E_{2 x} \times L_{2} \\
& D_{R}=\cos E_{3 x} \times L_{3}+\cos E_{4 x} \times L_{4}
\end{aligned}
$$

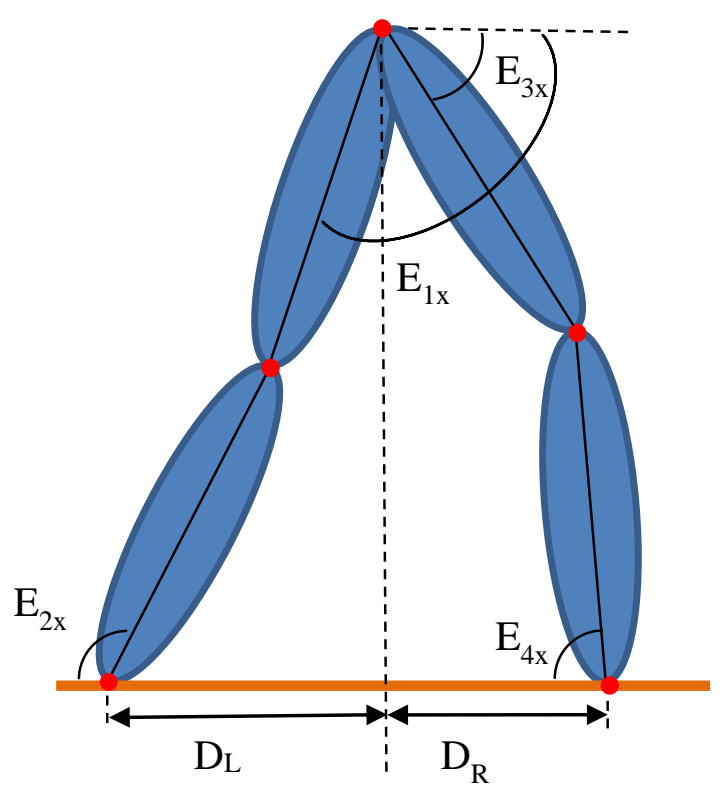

\section{Figure 13: Denotations of Euler angles and relative displacements along primary direction, viewed from the side of pedestrian}

However this calculation does not include possible sideway inclinations of the legs. The legs might not be confined to the plane defined by the gravity and the walking direction, as shown in Figure 13. More often than not, the legs stretch sideway while 
walking or standing, as depicted in Figure 14. The step length along the primary walking direction is thus needed to be computed from the actual leg lengths' projections. The actions of side-stepping and going on stairs are not considered in MotionTracker, so there are no calculations for displacements of the two feet along any directions other than front and back.

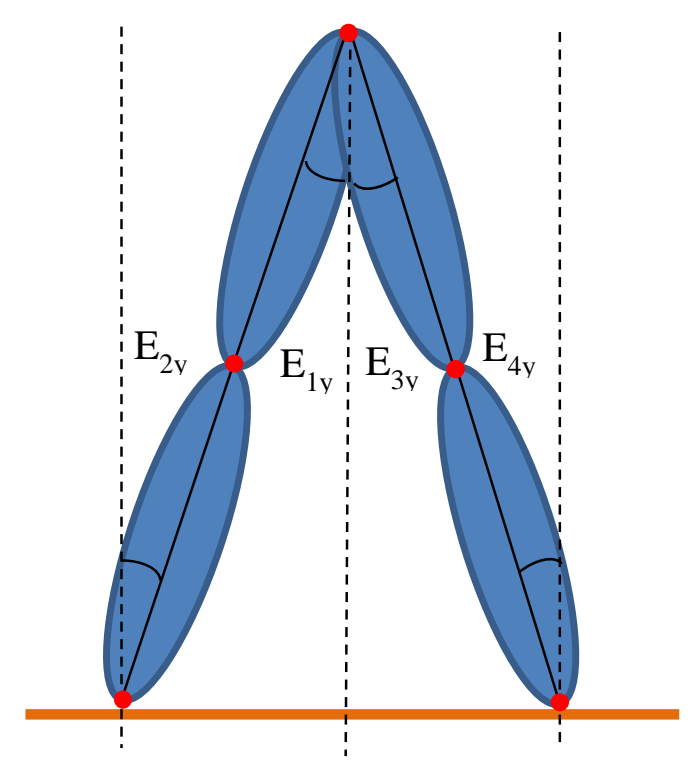

Figure 14: Denotation of Euler angles, viewed from the back of pedestrian

Hence the new formulas that consider this effect is presented below:

$$
\begin{aligned}
& D_{L}=\cos E_{1 y} \times \cos E_{1 x} \times L_{1}+\cos E_{2 y} \times \cos E_{2 x} \times L_{2} \\
& D_{R}=\cos E_{3 y} \times \cos E_{3 x} \times L_{3}+\cos E_{4 y} \times \cos E_{4 x} \times L_{4}
\end{aligned}
$$

By comparing the two values, it is possible to determine which foot is at front, and the difference with the other foot is the step length at that moment. With the provided information, it may be possible to find out whether the pedestrian is walking forward or backward; but this would involve analyzing all the angles carefully through a step and knowledge in human walking pattern, which is beyond the scope of this thesis. For 
MotionTracker, the assumption of a pedestrian always walks forward is made. Therefore only the step length $\mathrm{S}$ is needed to calculate the walking path of the pedestrian. For the instance in Figure 13 the step length can then be calculated as follows:

$$
\mathrm{S}=\left|D_{R}-D_{L}\right|
$$

However this step length could not be directly used for accumulation to get the total distance travelled by a pedestrian. In fact, the sensor data can come at any time during a step and it is not guaranteed to be the moment when both feet are on the ground. This problem could be resolved by using a 5th sensor as pedometer to count the step. Alternatively, another method is used to determine when a step is finished.

\subsection{How to differentiate between steps}

It can be inferred that for normal walking biomechanics, the actual step length is closest to the maximum step length recorded in a step's time. To find the exact moment a step is finished, is equivalent to find the moment $D_{R}$ becomes larger than $D_{L}$ or vice versa. However, because of the presence of noises, this might happen multiple times during a step, especially when the data rate is high. To avoid noises causing the system to miscount the steps, a step threshold, denoted as Es, needs to be applied. Es is determined by the error in angles and limb length. Only when the step length S (the absolute difference between $D_{R}$ and $D_{L}$ ) is greater than the threshold $E_{S}$, the system can conclude that the pedestrian is really making a step. To determine the boundary between multiple steps made by the pedestrian, it is also vitally important to detect which foot is at front. Consider the normal walking pattern, both feet alternate to be in the front. Hence in our system, a new step only happens if the other foot comes to the front, while the step length $\mathrm{S}$ is greater than the threshold Es.

The standing situation also needs to be considered. When the pedestrian is standing still, the two feet should be close to each other and the step length $S$ should be constantly around 0 . Hence the program understands that the pedestrian is standing when the step length $\mathrm{S}$ is consistently less than the threshold Es for an extended period of time. In this case there is not a single feet that is at the front. When the pedestrian starts to walk, the 
step length $\mathrm{S}$ becomes greater than the threshold Es. At this moment the program needs to find out the feet at front for the first step, and set the state to walking state. When the pedestrian stops walking to stand still, the program needs to check the step length for a number of iterations based on the data rate. This is done by defining a count $\mathrm{K}_{\mathrm{S}}$, which is incremented for every iteration with the step length $\mathrm{S}$ less than the threshold Es. If $\mathrm{K}_{\mathrm{S}}$ becomes equal or greater than a constant number $\mathrm{C}$, the current step is finished and the state is set to standing state. The constant $\mathrm{C}$ should be dependent on the data rate, allowing the program to detect the standing mode in the timeframe of 1 or 2 seconds.

Table 7 shows the complete state machine for the MotionTracker system.

Table 7: The state machine for MotionTracker

\begin{tabular}{|c|c|c|c|}
\hline Current State & Condition & Next State & Effect \\
\hline Standing State & $\mathrm{S}<$ Es & Standing State & \\
\hline Standing State & $\mathrm{S}>$ Es and $\mathrm{D}_{\mathrm{L}}>\mathrm{D}_{\mathrm{R}}$ & $\begin{array}{l}\text { Walking State, } \\
\text { Left }\end{array}$ & Start a new step \\
\hline Standing State & $\mathrm{S}>$ Es and $\mathrm{D}_{\mathrm{R}}>\mathrm{D}_{\mathrm{L}}$ & $\begin{array}{l}\text { Walking State, } \\
\text { Right }\end{array}$ & Start a new step \\
\hline $\begin{array}{l}\text { Walking State, } \\
\text { Left }\end{array}$ & $\mathrm{S}>$ Es and $\mathrm{D}_{\mathrm{L}}>\mathrm{D}_{\mathrm{R}}$ & $\begin{array}{l}\text { Walking State, } \\
\text { Left }\end{array}$ & reset $\mathrm{K}_{\mathrm{S}}$ \\
\hline $\begin{array}{l}\text { Walking State, } \\
\text { Left }\end{array}$ & $\mathrm{S}>$ Es and $\mathrm{D}_{\mathrm{R}}>\mathrm{D}_{\mathrm{L}}$ & $\begin{array}{l}\text { Walking State, } \\
\text { Right }\end{array}$ & $\begin{array}{l}\text { Finish current step, start } \\
\text { a new step, reset } K_{S}\end{array}$ \\
\hline $\begin{array}{l}\text { Walking State, } \\
\text { Left }\end{array}$ & $\mathrm{S}<$ Es and $\mathrm{K}_{\mathrm{S}}<\mathrm{C}$ & $\begin{array}{l}\text { Walking State, } \\
\text { Left }\end{array}$ & Increment $\mathrm{K}_{\mathrm{S}}$ \\
\hline $\begin{array}{l}\text { Walking State, } \\
\text { Left }\end{array}$ & $\mathrm{S}<\mathrm{Es}$ and $\mathrm{K}_{\mathrm{S}}>\mathrm{C}$ & Standing State & $\begin{array}{l}\text { Finish current step, } \\
\text { reset } K_{S}\end{array}$ \\
\hline
\end{tabular}




\begin{tabular}{|l|l|l|l|}
\hline $\begin{array}{l}\text { Walking State, } \\
\text { Right }\end{array}$ & $\mathrm{S}>$ Es and $\mathrm{D}_{\mathrm{L}}>\mathrm{D}_{\mathrm{R}}$ & $\begin{array}{l}\text { Walking State, } \\
\text { Left }\end{array}$ & $\begin{array}{l}\text { Finish current step, start } \\
\text { a new step, reset } \mathrm{K}_{\mathrm{S}}\end{array}$ \\
\hline $\begin{array}{l}\text { Walking State, } \\
\text { Right }\end{array}$ & $\mathrm{S}>$ Es and $\mathrm{D}_{\mathrm{R}}>\mathrm{D}_{\mathrm{L}}$ & $\begin{array}{l}\text { Walking State, } \\
\text { Right }\end{array}$ & reset $\mathrm{K}_{\mathrm{S}}$ \\
\hline $\begin{array}{l}\text { Walking State, } \\
\text { Right }\end{array}$ & $\mathrm{S}<$ Es and $\mathrm{K}_{\mathrm{S}}<\mathrm{C}$ & $\begin{array}{l}\text { Walking State, } \\
\text { Right }\end{array}$ & Increment $\mathrm{K}_{\mathrm{S}}$ \\
\hline $\begin{array}{l}\text { Walking State, } \\
\text { Right }\end{array}$ & $\mathrm{S}<$ Es and $\mathrm{K}_{\mathrm{S}}>\mathrm{C}$ & Standing State & $\begin{array}{l}\text { Finish current step, } \\
\text { reset } \mathrm{K}_{\mathrm{S}}\end{array}$ \\
\hline
\end{tabular}

The rare situation in which the pedestrian stops in the middle of a step with two feet separated is not considered here. This situation is somewhat covered in the current state machine. Since the step length does not close, the step does not finish, and the current recorded maximum step length will be kept for the whole duration of the stop. As the pedestrian starts walking again the final step length could be determined as the step finishes.

The step length of a step is only recorded when the step is finished. At this moment, the current maximum step length over the entire duration of the step become the recorded step length of this step, and is thus added to the total distance walked by the pedestrian.

\subsection{Android app design}

In the MotionTracker system, the remote data processing device is developed on Android platform. The MotionTracker Android app handles the Bluetooth Smart communications similar to the example app provided by Texas Instrument for its SensorTag [20]. The service that controls connections from the example app is retrofitted to allow multiple sensor nodes connecting to one Android device at the same time. When connections are established, the MotionTracker app receives continuous updates of Euler angles, calculates the step lengths and total distances walked, and displays the results to the user. 
The app starts with the scanning interface where all broadcasting sensor nodes of the system are listed and available for connection, while other BLE devices are filtered. The user could touch the listed sensor to establish connection. Once all the connections are established, a button can be pressed to start the system. The app enters the interface that display Euler angles sent from all the sensor nodes. At the same time, the app commands the sensor nodes to send notifications when new data are available. A broadcast receiver handles all interrupts from the BLE module on the smartphone and invokes actions when new data arrive from the sensor nodes. These actions include updating the interface with the newest values and analysis results.

Before actually calculating the step length, the app needs to pair the sensor nodes with the locations they are attached to, and load the lengths of limb. These parings and lengths of limb have to be given by a user. The user could change these settings in the preferences.

After these preparations the app is ready to start recording and calculating the how pedestrian walks. At the start, the system is initialized into standing mode. Then, as described in sections 4.1 and 4.2, the app enters a state machine to count the steps, determine the step length, and eventually calculate the total distance walked by a user. These results are reported to the user interface of the app on the run.

The source code and the instructions to load the program to an Android device can be found in Appendix C. 


\section{Chapter 5: Experiments and Results}

In this chapter the results of the inertial sensor network developed in previous chapters is reported. In section 5.1 the experiment method to test an individual sensor node is presented and the results are analyzed. The superior performance of the inertial sensor network as well as its limitations are discussed in detail in section 5.2. Finally section 5.3 shows the results of the example application MotionTracker.

\subsection{Performance of individual sensor node}

To evaluate the performance of an individual sensor node, experiments must be set up to test the different performance metrics of the system: data accuracy, throughput, timing, and latency. These performance indicators are very important to the inertial sensor network as a whole since they set the limits for applications developed for the network.

\subsubsection{Data accuracy at rest}

To determine the data accuracy of the sensor node at rest, the zero biases, drifts, and noises of all the sensor outputs need to be measured. Experiments are designed to keep a sensor node at rest for several minutes on a horizontal surface to record sensor readings from accelerometer, gyroscope, and magnetometer, plus Euler angles results from the DCM algorithm. There should be no external forces applying to the sensor node except the gravity during the experiments. These experiments are conducted by setting one of the sensor nodes on a desk for 2 to 5 minutes. The sensor node is placed such that the accelerations on the horizontal axes are minimized to make the sensor node board as close to horizontal as possible. With this arrangement, the $\mathrm{x}$ axis and $\mathrm{y}$ axis of the sensor node are roughly horizontal and the $\mathrm{z}$ axis largely follows the direction of the gravity. Although the sensor node is not screwed to the desk, during the experiment it is left untouched and best efforts are made to ensure it is not moving in any way. The sensor node is set to report one type of data at $40 \mathrm{~Hz}$ through ICDI debug interface to a PC for each experiment. A stopwatch is used to ensure the data are measured at the correct frequency, by comparing the number of data sets measured and time elapsed. 
Figure 15 shows the raw readings (unit: $\mathrm{m} / \mathrm{s}^{2}$ ) for all 3 axes of accelerometer. The vertical scale represents the accelerometer readings while the horizontal scale represents the enumeration of samples for all graphs.

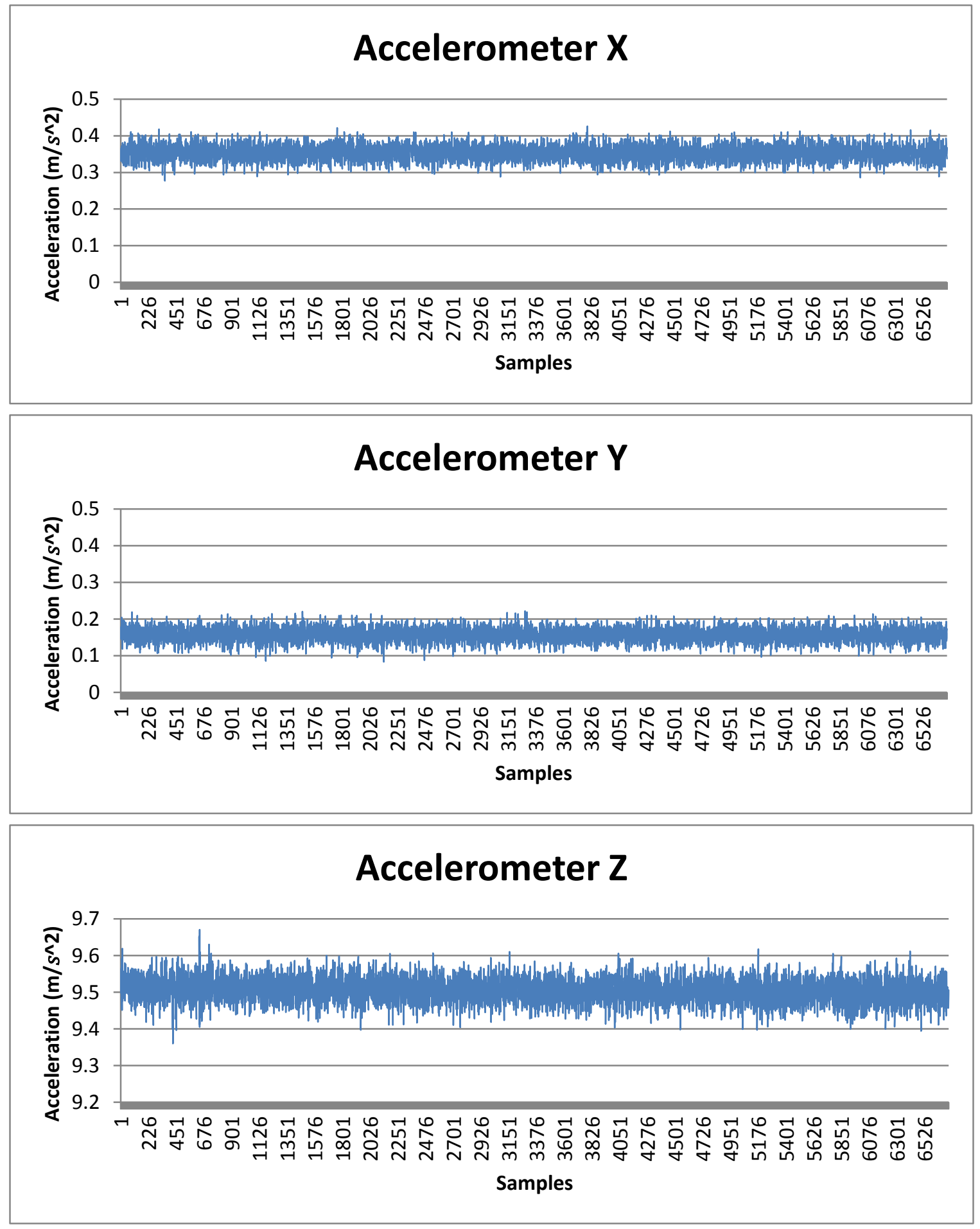

Figure 15: The accelerometer results for Sensor Node 2 when at rest on desk surface 
After analyzing the raw data in Figure 15, the averages, standard deviations, noises and the slopes of the readings for each axis are computed and presented in Table 8 . The slope for all axes are close to 0 , indicating that the accelerometer results do not drift over time. The noises data are calculated according to the following noise spectral density formula:

$$
N_{0}=\frac{\sigma}{\sqrt{B W}}
$$

In this formula, $\sigma$ represents the standard deviation of the data, while BW represents the frequency of the data. The noise density calculated for the $\mathrm{x}$ and $\mathrm{y}$ axes largely agree with the noise density data in the specifications of the MPU9150 sensor $(400 \mu \mathrm{g} / \sqrt{\mathrm{Hz}})$. Obviously, as depicted in Figure 15, the $\mathrm{z}$ axis is significantly noisier with a noise density of $533.3 \mu \mathrm{g} / \sqrt{\mathrm{Hz}}$. This is due to the physical structure of 3 -axis accelerometer in the MPU9150 chip. Often, for the horizontal axes, $x$ and $y$, there are enough space in the package to put metal beams that change capacitance when forces are applied along $\mathrm{x}$ and $\mathrm{y}$ directions. For the $\mathrm{z}$ axis, the package is not high enough to allow the same structure as $\mathrm{x}$ and $\mathrm{y}$. Instead, a cantilever system is used, also changing the capacitance when forces are applied along $\mathrm{z}$ axis. This structure, however, is usually noisier than the simple metal beam structure. Therefore it is common that for many accelerometers, the $\mathrm{z}$ axis has more noises than the $\mathrm{x}$ and $\mathrm{y}$ axes.

Table 8: The acceleration data for Sensor Node 2 when at rest on desk surface

\begin{tabular}{|l|c|c|c|}
\hline & $\mathrm{X}$ & $\mathrm{Y}$ & $\mathrm{Z}$ \\
\hline Average $\left(\mathrm{m} / \mathrm{s}^{2}\right)$ & 0.3530 & 0.1563 & 9.496 \\
\hline $\begin{array}{l}\text { Standard deviation } \\
\left(\mathrm{m} / \mathrm{s}^{2}\right)\end{array}$ & 0.02061 & 0.01871 & 0.03305 \\
\hline Noises $(\mu \mathrm{g} / \sqrt{\mathrm{Hz}})$ & 332.5 & 301.9 & 533.3 \\
\hline Slope & $3.149 \mathrm{E}-09$ & $-3.723 \mathrm{E}-08$ & $-1.063 \mathrm{E}-06$ \\
\hline
\end{tabular}


Table 8 also shows that there exists a quite significant amount of zero biases and possibly some linear biases in the accelerometer readings. The total acceleration, by combining the accelerations from all 3 axes, is calculated to be $9.504 \mathrm{~m} / \mathrm{s}^{2}$, as opposed to the gravity constant of approximately $9.81 \mathrm{~m} / \mathrm{s}^{2}$. Experiments on other sensor nodes have yielded similar results. Therefore when using the acceleration data it is important to calibrate the sensor node to remove zero biases and linear biases. Another error contributing to these results is the tilt between the $\mathrm{z}$ axis and the direction of gravity. However there is no way to perfectly place the sensor node so that the $\mathrm{z}$ axis could match the direction of gravity exactly, hence there is always a small component of the gravity in the $\mathrm{x}$ and $\mathrm{y}$ axes. Thus it is only possible to estimate the zero biases with the assumption that the tilt is 0 .

The gyroscope data appears to have better quality than the accelerometer data, as shown in Figure 16, with very small range of fluctuation. And all 3 axes of the gyroscope have equally good results. Same statistic values as accelerometer are calculated for gyroscope measurements; they are shown below in Table 9. These data have much less noises than the accelerometer, even though the numeric range is broader $\left(250^{\circ} / \mathrm{s} \mathrm{vs} 2 \mathrm{~g}\right)$. Similar to the accelerometer, there is no drift for the sensor since the slope is negligible. As the sensor node is at rest, all the non-zero data are actually the zero-biases. The averages of readings for different axes show that the $\mathrm{x}$ axis has a bigger zero bias than the $\mathrm{y}$ and $\mathrm{z}$ axes. The existence of these zero biases, even though small, could still cause the results to diverge after integration. Thus when using the raw gyroscope data, these values should be deducted from the sensor outputs. 

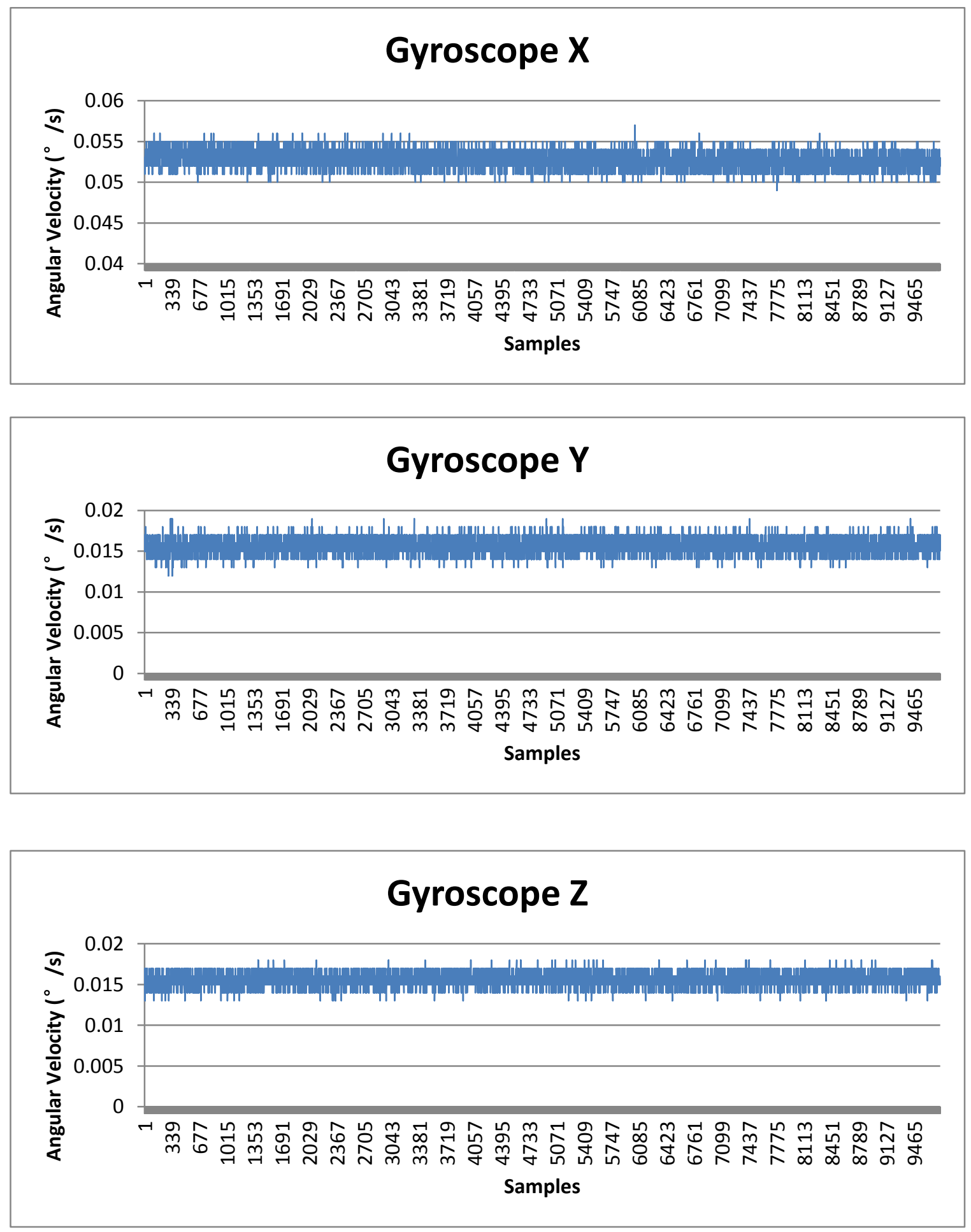

Figure 16: The gyroscope data for Sensor Node 2 when at rest on desk surface 
Table 9: The gyroscope data for Sensor Node 2 when at rest on desk surface

\begin{tabular}{|l|c|c|c|}
\hline & $\mathrm{X}$ & $\mathrm{Y}$ & $\mathrm{Z}$ \\
\hline Average $(\% / \mathrm{s})$ & 0.05262 & 0.01556 & 0.01570 \\
\hline $\begin{array}{l}\text { Standard deviation } \\
(\% / \mathrm{s})\end{array}$ & 0.0009901 & 0.0009297 & 0.0008129 \\
\hline Noises $(\% / \mathrm{s} / \sqrt{\mathrm{Hz}})$ & 0.0001565 & 0.0001470 & 0.0001285 \\
\hline Slope & $-2.965 \mathrm{E}-08$ & $-1.398 \mathrm{E}-08$ & $1.912 \mathrm{E}-08$ \\
\hline
\end{tabular}

The magnetometer measurements have been conducted in a typical home environment where electronic devices are present. Table 10 summarizes the magnetometer readings in statistics.

Table 10: The Magnetometer results for Sensor Node 2 when at rest on desk surface

\begin{tabular}{|l|c|c|c|}
\hline & $\mathrm{X}$ & $\mathrm{Y}$ & $\mathrm{Z}$ \\
\hline Average $(\mu \mathrm{T})$ & -8.416 & 22.43 & 49.44 \\
\hline $\begin{array}{l}\text { Standard deviation } \\
(\mu \mathrm{T})\end{array}$ & 0.8335 & 0.8296 & 0.8153 \\
\hline Noises $(\mu \mathrm{T} / \sqrt{\mathrm{Hz}})$ & 0.1318 & 0.1312 & 0.1289 \\
\hline Slope & $-4.655 \mathrm{E}-06$ & $-8.842 \mathrm{E}-06$ & $-9.033 \mathrm{E}-06$ \\
\hline
\end{tabular}

Magnetometer data in Table 10 show that the magnetic field measurement are quite stable when the sensor node is at rest and no major interference is near the sensor. The slope is close to 0 and the noises are small compared to the magnitude of average readings. 
To evaluate the magnetometer's accuracy, the sensor node is turned around on a horizontal surface to find out the max and min values for $\mathrm{x}$ and $\mathrm{y}$ axes. It turns out that the y axis value for Sensor Node 2 changes from close to 0 to more than 50, but never becomes negative, while the $\mathrm{x}$ axis value measures more negative values than positive values. This indicates that the magnetometer has significant zero biases. In the rotation of the sensor node, the $\mathrm{z}$ axis stays roughly the same most of the time, as expected for horizontal movements. The conclusion is that the magnetometer of the sensor node cannot be trusted for calculating direction on its own in home and office environment. The magnetometer should always be used with other sensors to compute a more reliable heading.

An experiment to evaluate the accuracy of the Euler angle output of the sensor node is also conducted. The Euler angles are calculated from measurements of accelerometer, gyroscope, and magnetometer, hence the accuracy of the Euler angles largely depend on the data input to the DCM algorithm. Since the accelerometer and gyroscope data both demonstrate good accuracy at rest, the roll and pitch angles that depend on these results are expected to be accurate. The yaw (heading) data, on the other hand, largely depend on the magnetometer, which is proven to be unreliable. Therefore if the DCM algorithm has reasonable performance, the roll and pitch data should be relatively accurate while the yaw data may have more errors. Figure 17 shows the roll, pitch, and yaw angles of the Euler angle output of the sensor node. The vertical scale shows the angles in degrees. 


\section{Euler Angle X (Roll)}
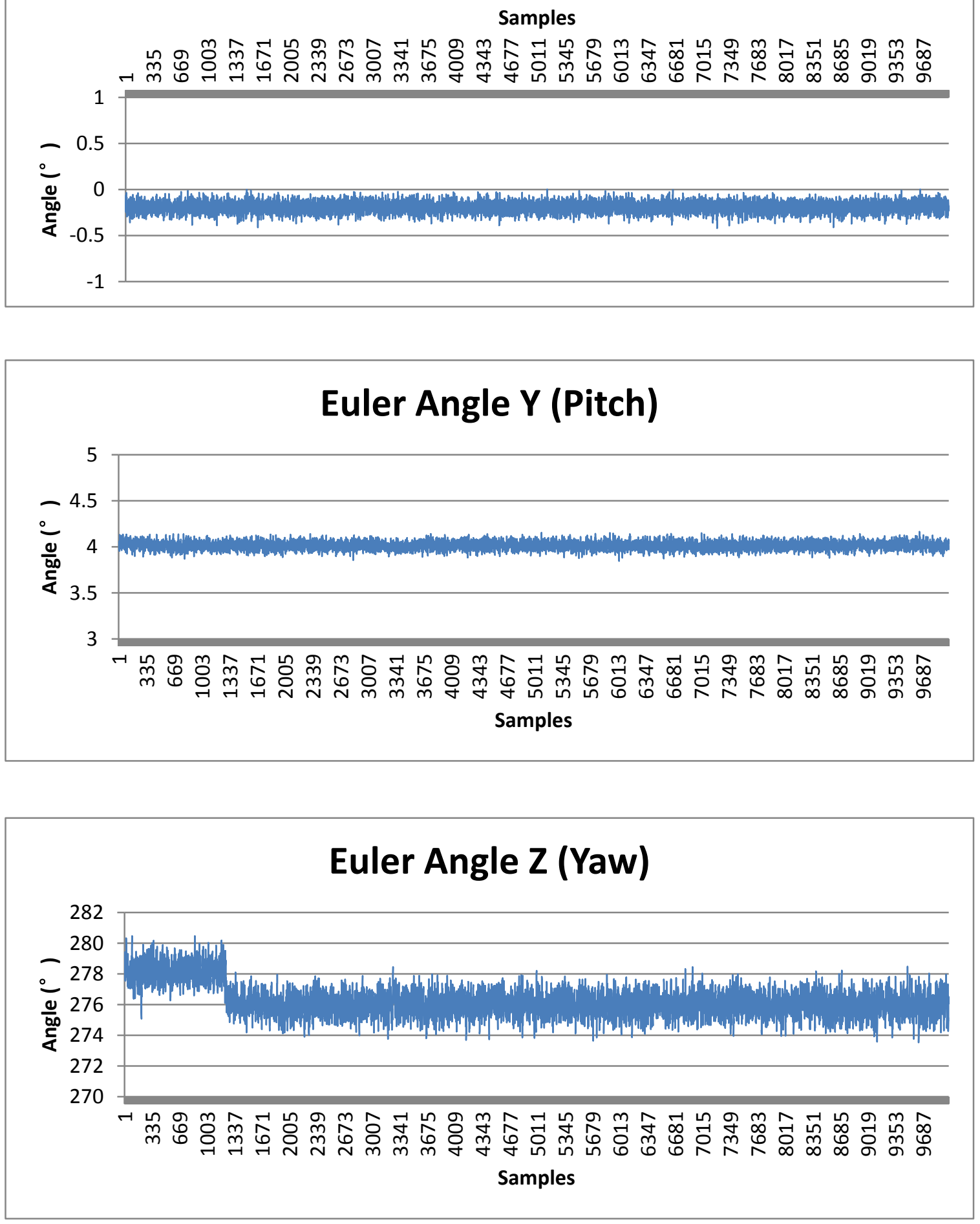

Figure 17: The Euler angle data for Sensor Node 2 when at rest on desk surface 
As Figure 17 shows, the roll and pitch angles are relatively stable, while the yaw angle has a huge shift at around the $1000^{\text {th }}$ sample. This is probably due to yaw angle is almost solely computed with results from magnetometer when the sensor is stationary. The MPU9150's magnetometer could suffer from various magnetic disturbances commonly found in home and office environment. This is probably one of cases that the magnetic field changes in the environment since data in pitch and roll do not seem to indicate a movement of the sensor node itself at the exact moment.

The statistics of the Euler angle results are presented in Table 11.

Table 11: The Euler Angle results for Sensor Node 2 when at rest on desk surface

\begin{tabular}{|l|c|c|c|}
\hline & X (Roll) & Y (Pitch) & Z (Yaw) \\
\hline Average $\left(^{\circ}\right)$ & -0.1935 & 4.012 & 276.1 \\
\hline Standard deviation $\left(^{\circ}\right)$ & 0.05633 & 0.04215 & 0.9033 \\
\hline Slope & $-1.603 \mathrm{E}-07$ & $-4.692 \mathrm{E}-07$ & $-3.714 \mathrm{E}-05$ \\
\hline
\end{tabular}

Table 11 demonstrates the results computed by the DCM library provided by Texas Instruments. The library function takes input from the raw accelerometer, gyroscope, and magnetometer readings, without any filtering. The low standard deviations of roll and pitch suggest that there are little noises in these results. However the yaw angle produces much greater standard deviation than the other axis, because of the shift at around the $1000^{\text {th }}$ sample. If the first 2000 samples are truncated, the yaw angle would have an average angle of $276.0^{\circ}$, a standard deviation of $0.6995^{\circ}$, and a slope of $-2.330 \mathrm{E}-06$. These results are still many times worse than the other axes.

\subsubsection{Data accuracy while moving}

Since the inertial network is designed to recognize or measure actions, it is important to understand how well the sensor nodes perform in a moving situation. To test this, one of the sensor nodes is mounted on a robotic arm with 6 degrees of freedom. In order to simulate consumer usage, the sensor node is only tightly fasten to the robotic arm using 
bandages, not permanently attached to it in any way. The sensor node is placed such that the $y$-axis of the sensor node goes along the direction of the arm from the base to the top; the $\mathrm{x}$-axis goes sideway, vertical to the robotic arm; the $\mathrm{z}$-axis goes into the robotic arm, vertical to its top surface. The experiment lets the robotic arm moving constantly between 45 and 90 degrees, and the sensor node measures the movement using Euler angles. A video camera is used to capture the movement. The results from the video camera are compared with the sensor node results. In order to match the sensor data with the video camera as closely as possible, the data rate for the sensor node is set at $50 \mathrm{~Hz}$ and the frame rate for the video camera is set at $48 \mathrm{~Hz}$. The experiment shows that the robotic arm overshoots a bit after reaching the target angle. Sometimes after overshooting the arm is visibly shaking back and forth around the target angle. Therefore after each movement, the robotic arm is programmed to wait for 5 seconds before moving back, so the arm could stabilize over the period. A comparison is made between the peak data recorded by the sensor node and the angle measured from the corresponding video frame. Similar comparison is done with the stabilized data and its corresponding video frame as well. 


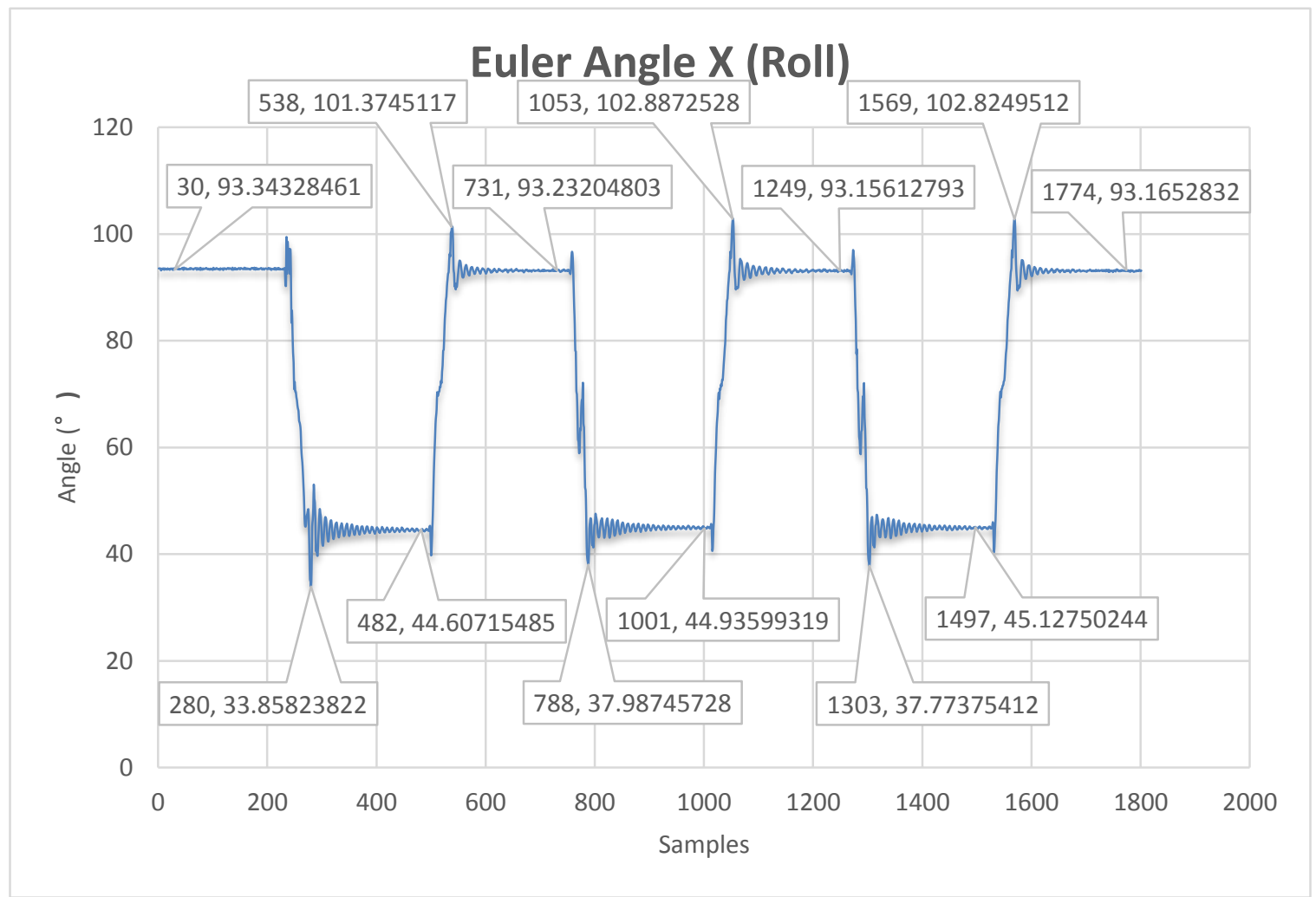

\section{Figure 18: Euler angle in $\mathrm{X}$ axis (roll, the main axis of motion) for robotic arm to move between 90 and 45 degrees}

In the experiment, the robotic arm moves from 90 degree position to 45 degree position and back 3 times. Figure 18 shows the primary Euler angle results produced by the sensor node attached to the robotic arm during this motion. The vertical scale shows angle in degrees. The labelled data points have the format of (sample number, angle in degrees). These angle values are compared to the measured angles from their corresponding video frame. The video is captured by a video camera from the side of the robotic arm. The original video has to go through a de-fisheye process to restore the scene. Since the video is captured at $48 \mathrm{~Hz}$, the frames do not have a 1-1 relation with the results measured by the sensor node. The corresponding frames are found by going through the video frame by frame and looking for the overshooting moments and the stabilized moments of the robotic arm. Even so, the frames are captured at slightly different times from their corresponding sensor measurements. But this difference should not have any significant effect on the comparison. 
Table 12 shows the comparison between the angle measured by the sensor node and "pictured angle", the angle captured by the video camera. By using "measure" tool in GIMP image processing software on the robotic arm and the platform, relative angle to the horizontal plane of the video frame can be found. The pictured angle is thus calculated as the difference of the angle of the robotic arm and the angle of the platform. The error is the difference between measured Euler angle around X-axis (roll) and the pictured angle.

Table 12: Comparison between the angle measured by the sensor node and the pictured angle on corresponding video frame (unit: degree)

\begin{tabular}{|c|c|c|c|}
\hline Motor 2 Angle $\left({ }^{\circ}\right)$ & Pictured Angle $\left(^{\circ}\right)$ & Measured Angle $X\left(^{\circ}\right)$ & Error $\left(^{\circ}\right)$ \\
\hline 90 & 94.66 & 93.34 & -1.317 \\
\hline 45- Overshoot & 43.82 & 33.86 & -9.962 \\
\hline 45 & 45.4 & 44.61 & -0.7929 \\
\hline 90+ Overshoot & 96.69 & 101.4 & 4.685 \\
\hline 90 & 94.36 & 93.23 & -1.128 \\
\hline 45- Overshoot & 45.54 & 37.99 & -7.553 \\
\hline 45 & 45.83 & 44.94 & -0.8940 \\
\hline 90+ Overshoot & 96.69 & 102.9 & 6.197 \\
\hline 90 & 94.42 & 93.16 & -1.264 \\
\hline 45- Overshoot & 45.69 & 37.77 & -7.916 \\
\hline 45 & 45.83 & 45.13 & -0.7025 \\
\hline 90+ Overshoot & 96.69 & 102.8 & 6.135 \\
\hline
\end{tabular}




\begin{tabular}{|l|l|l|l|}
\hline 90 & 94.22 & 93.17 & -1.055 \\
\hline Average Error & & & 0.007238 \\
\hline $\begin{array}{l}\text { Standard Deviation } \\
\text { Error }\end{array}$ & & & 4.921 \\
\hline
\end{tabular}

In Table 12, the angles measured by the sensor node and by the video frame are compared in different situations. Each row of the data is corresponding to a data tag in Figure 18 in sequence. It is obvious that in overshooting situation, the absolute differences between two types of measurements increase significantly. Since the video captured angle more or less represents the actual angle the sensor node should be experiencing, the results indicate that the sensor node performs poorly in measuring Euler angles when the motion is abrupt, probably due to high acceleration at the moment. This is essentially a problem with the DCM algorithm and the weights of the algorithm for accelerometer, gyroscope, and magnetometer. Aside from the overshooting data points, the sensor node shows consistency in the other data points, with an error around -1 degree. This constant error is due to the positioning of the senor on the robotic arm and the biases in measuring the angle from video frames. This means the sensor could measure Euler angles in good accuracy and consistency if the motion is slow and gentle.

The Euler angle results in y-axis (pitch), and z-axis (yaw) are presented in Figure 19 and Figure 20 below. 


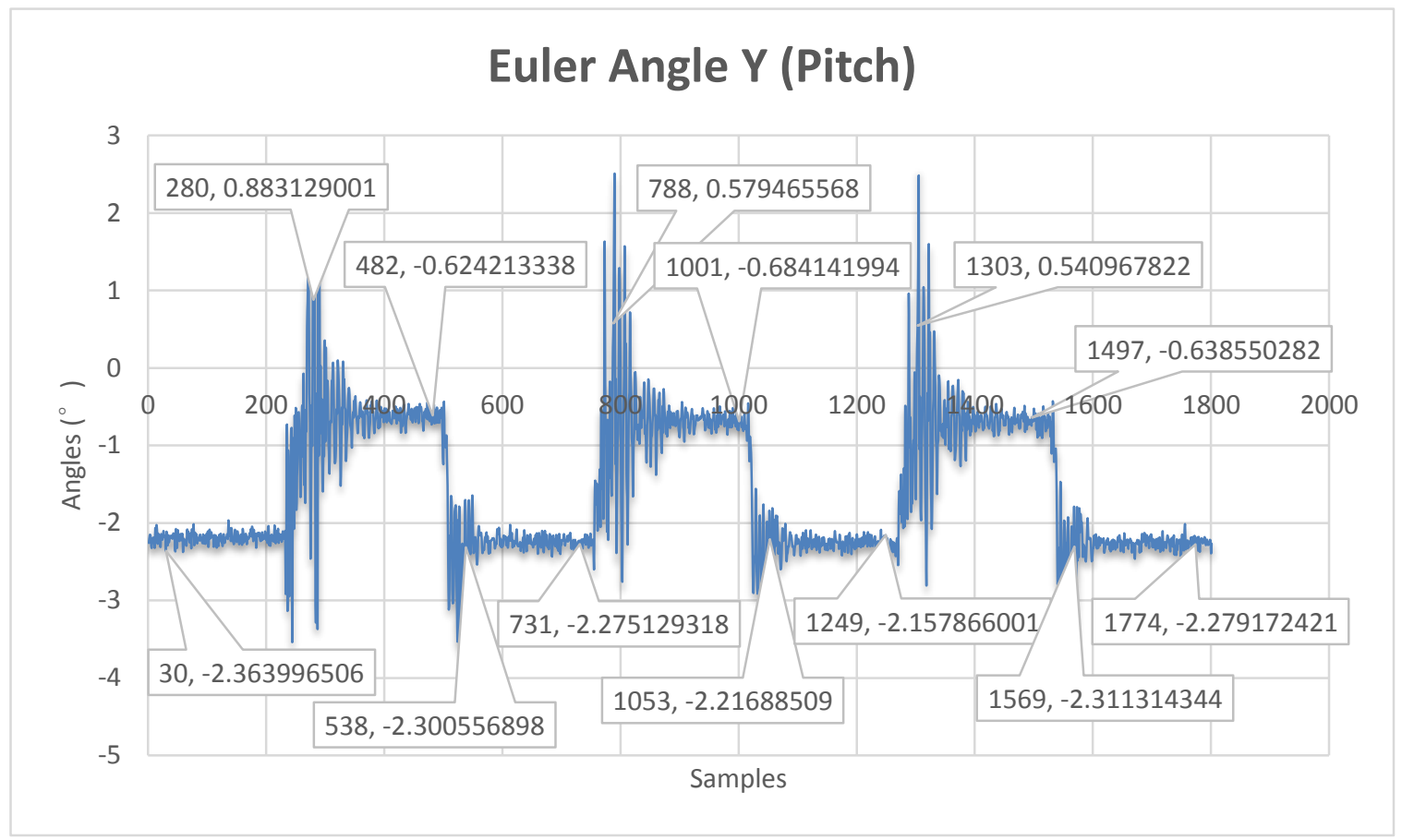

Figure 19: Euler angle in $Y$ axis (pitch, off-motion axis)

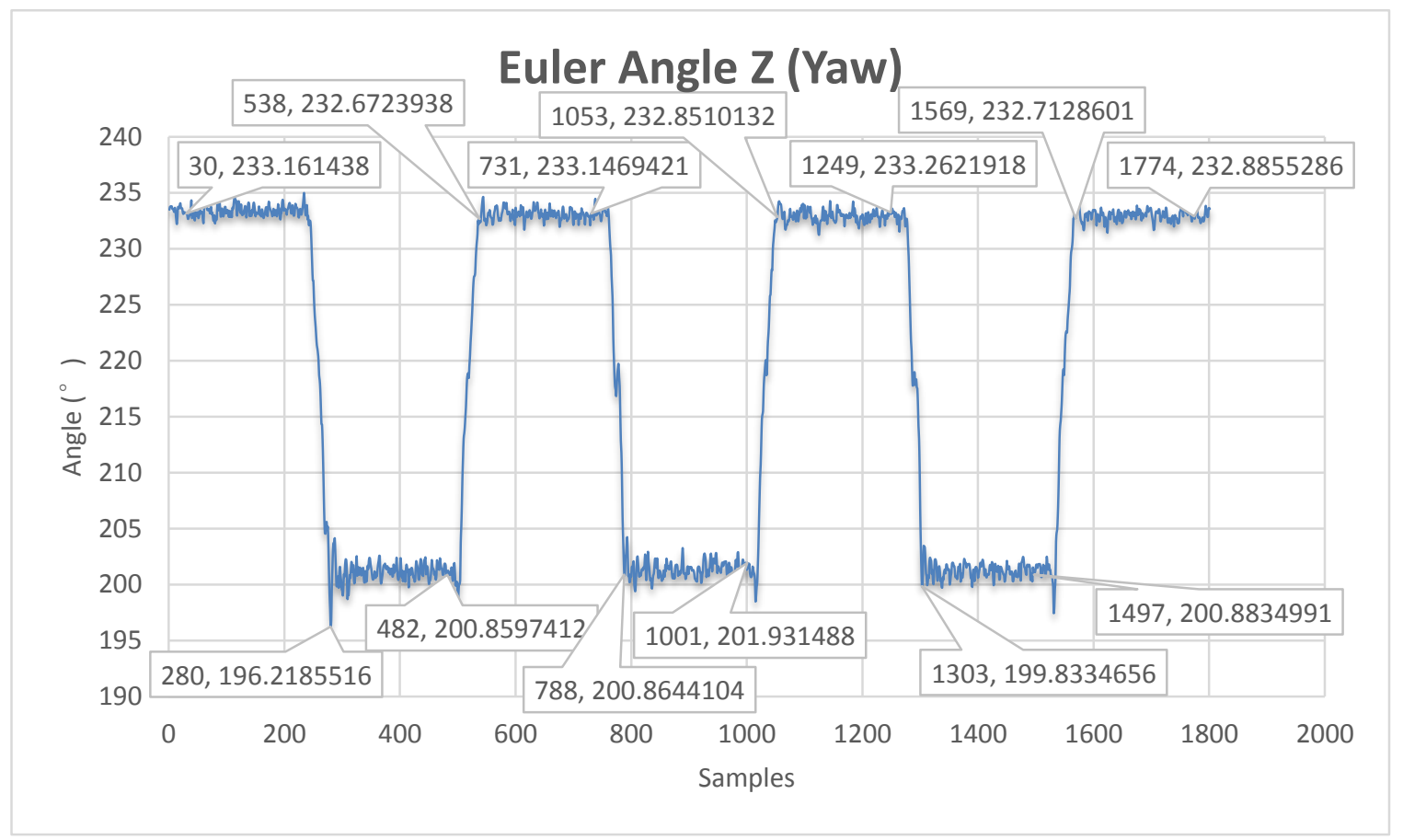

Figure 20: Euler angle in $\mathrm{Z}$ axis (yaw, off-motion axis) 
Results in Figure 19 and Figure 20 suggest that, as the robot arm goes from 90 degree to 45 degree, the pitch and yaw (heading) angles change at about the same pace as roll angle. However, the Euler angle measurements of yaw are quite inaccurate as shown in sub-section 5.1.1, so it is only reliable to look at roll and pitch. It should be noted that in Figure 19 the pitch angle results seem to have a lot more noises than roll and yaw when the robot arm is at move, the truth is the vertical scale of Figure 19 is much smaller than the other two figures and all axes should have similar level of noises while the sensor node is at move. The small changes of the pitch angle between 90-degree and 45-degree position may represent the actual changes of the sensor orientation, since both values are quite close to 0 . However, without another form of measurement, it is impossible to tell how much errors are there in the pitch angle measurements. The only conclusion is that both pitch and roll angle have less noise when the robotic arm is not moving violently.

\subsubsection{Data rate limitation for BLE}

A sensor node has a limitation on the throughput of the sensor data over Bluetooth Smart. This limitation is found when the receiving end could no longer receive as many data packets as the sensor node collects from MPU9150. An experiment has been carried out to determine the maximum data frequency of transmission between a single sensor node and an Android device, a Galaxy Nexus smartphone. The sensor node is set to transmit 12 bytes of data together in one write command every cycle. The 12 bytes of data is constructed as a counter to count from 0 to 359 iteratively. When the Android device receives a set of data from the sensor node, it records the time. After 5 minutes, there is a long list of timestamps corresponding to the data. The periods between transmissions can be found by subtracting the neighboring timestamps. Table 13 summarizes the statistics of the time periods between the arrivals of data packets.

Table 13: Periods between transmissions at receiving end for different data transmission rate

\begin{tabular}{|l|l|l|l|}
\hline Data rate $(\mathrm{Hz})$ & 100 & 200 & 250 \\
\hline
\end{tabular}




\begin{tabular}{|l|c|c|c|}
\hline $\begin{array}{l}\text { Average period } \\
(\mathrm{ms})\end{array}$ & 9.996 & 4.996 & 4.477 \\
\hline $\begin{array}{l}\text { Standard deviation } \\
\text { of period (ms) }\end{array}$ & 8.007 & 5.487 & 5.029 \\
\hline
\end{tabular}

As Table 13 shows, the results are exceptionally good. Even at $200 \mathrm{~Hz}$, the Android device is able to pick up every single transmission over the whole experiment. The average period is not exactly the same as $5 \mathrm{~ms}$ for $200 \mathrm{~Hz}$ experiment, because of the slightly different clock between the sensor node and the smartphone. At $250 \mathrm{~Hz}$, the average period $4.477 \mathrm{~ms}$ is more than $20 \%$ larger than the expected period of $4 \mathrm{~ms}$, indicating that many samples are lost in transmission. Indeed, after checking the data received, it is shown that 632 out of more than 60000 recordings are not consecutive with previous data. This means there are at least 632 cases that a minimum of one data packet is lost. If the data rate is pushed to $500 \mathrm{~Hz}$ (next frequency level) or more, the sensor node would run for a while, and then freeze because the UART channel is jammed.

In addition, the large standard deviations also suggest that the data packets are not arriving in uniform periods. By examining the data closely, it is found that most of the time data packets are arriving at 1 or 2 milliseconds intervals, but occasionally there are no data arriving for a large interval of nearly $100 \mathrm{~ms}$. This means the time recorded when the data packet arrives should not be used as if it is the actual time the data is collected.

The data rate limitation not only applies to the sensor node, but also to the device that is receiving the data packets. It is found that, for $100 \mathrm{~Hz}$ and $200 \mathrm{~Hz}$ experiments, even though there are no missing data packets with a Galaxy Nexus, there are many missing packets with an Oneplus One. In fact, it is found that the Oneplus One is only able to receive all packets if the data transfer rate is below $50 \mathrm{~Hz}$. Due to the varying specifications of consumer electronic devices, there is not a definite maximum working frequency for the sensor node. Therefore a speed test is needed for all applications to determine the maximum working data rate for any consumer devices. 


\subsubsection{Battery Life}

It is found that a single $3.3 \mathrm{~V}$ rechargeable coin battery of common brand could not power the entire sensor node board. The primary reason is likely that the battery is unable to keep the voltage at exactly $3.3 \mathrm{~V}$ which is required by the microcontroller. The solution of this problem is to use more powerful battery pack with output voltage higher than 3.3V. The battery pack should be connected to the sensor node power system before the regulator, so the voltage could be adjusted to exactly $3.3 \mathrm{~V}$. A control circuit is needed for the battery pack to be able to recharge when the sensor node is connected to a power source using micro-USB cable.

\subsection{Performance of inertial sensor network}

When combining many sensor nodes into an inertial sensor network, these sensor nodes need to connect with one single remote data processing device simultaneously. The most important performance metric for the sensor network as a whole is the data throughput, which is often limited by the implementation of the remote data processing device. Normally, the more sensor nodes are connected, the less frequently each sensor node is able to transmit the data due to congestion at the receiving end. In this experiment, 4 sensor nodes are connected to a Galaxy Nexus and every one of them is transmitting 12 bytes of data by BLE each cycle. The experiment runs for more than 10 minutes; it is set up in the same way as the experiment in sub-section 5.1.3. Whenever a data packet arrives, the Galaxy Nexus records the timestamp as well as its origin node. Table 14 shows the statistics of the periods between data packets from each sensor node.

Table 14: Transmission rate measured for 4 sensor nodes at receiving end

\begin{tabular}{|l|c|r|r|r|}
\hline & Node 1 & Node 2 & Node 3 & \multicolumn{1}{|l|}{ Node 4 } \\
\hline $\begin{array}{l}\text { Average period (ms) } \\
\text { at } 20 \mathrm{~Hz}\end{array}$ & 49.95 & 50.02 & 50.00 & 50.01 \\
\hline $\begin{array}{l}\text { Standard deviation } \\
\text { period (ms) at } 20 \mathrm{~Hz}\end{array}$ & 18.83 & 18.47 & 22.39 & 26.01 \\
\hline
\end{tabular}




\begin{tabular}{|l|c|c|c|c|}
\hline $\begin{array}{l}\text { Average period (ms) } \\
\text { at } 40 \mathrm{~Hz}\end{array}$ & 24.96 & 25.01 & 25.00 & 25.00 \\
\hline $\begin{array}{l}\text { Standard deviation } \\
\text { period (ms) at } 40 \mathrm{~Hz}\end{array}$ & 25.50 & 26.07 & 28.22 & 27.95 \\
\hline $\begin{array}{l}\text { Average period (ms) } \\
\text { at } 50 \mathrm{~Hz}\end{array}$ & 33.84 & 30.87 & 20.79 & 20.00 \\
\hline $\begin{array}{l}\text { Standard deviation } \\
\text { period (ms) at } 50 \mathrm{~Hz}\end{array}$ & 44.95 & 43.75 & 26.64 & 25.95 \\
\hline
\end{tabular}

As Table 14 shows, when there are 4 sensor nodes connecting to the Android device at the same time, the maximum working frequency is $40 \mathrm{~Hz}$. At this frequency and lower, the data packets from all sensor nodes are successfully received. For the $50 \mathrm{~Hz}$ case, data from Node 1 and Node 2 are coming at significantly larger intervals than the Sensor 3 and Sensor 4. That is because at some instances these data are coming in at a very high rate and the Galaxy Nexus is not able to handle them in time. Some data transmissions from Node 1 and Node 2 are completely lost. If the number of sensor nodes is reduced, then the system might not have this problem at the same frequency due to reduced workload for the receiving smartphone. But if the number of sensor nodes is to increase, then the maximum working frequency might be even lower than $40 \mathrm{~Hz}$.

\subsection{Results of MotionTracker PNS}

The major source of errors for measuring motion, as discussed in sub-section 5.1.2, is the overshoot of Euler angle results when the sensor is moving back and forth. Since the step length is calculated by using the biggest difference between the displacements of the two feet, it is guaranteed to suffer from overshoot errors. Although the overshoot error is near constant when the motion has a definite pattern, the pedestrian walking pattern is more random and therefore harder to estimate the error. Hence in the experiment the subject tries to be slow and gentle while stepping, so the overshoot error could be minimized. The sensor may also suffer from errors due to muscle movement during a 
step when attached to the legs. The biggest impact of this type error happens when the user is standing still, the error may cause the system to think the user has actually started walking. Therefore it is important to have a sufficiently large step threshold $E_{S}$ to prevent this problem.

The sensor nodes are placed in front of the subject's legs adjacent to the knees, keeping the $y$-axis aligned with the front surface of the legs towards top. There is an initial position error: the initial horizontal displacement of either foot may not equal to 0 . Since the step length takes the difference of the displacements, the error is mostly cancelled out, and only the error due to the difference in positioning of the sensors on two legs remains. Efforts are made to ensure the roll measurements from corresponding sensors on the two legs are as close to 0 as possible when the user is standing still.

The experiment is carried out indoor. The experiment is done with all sensor nodes powered by USB wires connecting to a PC, so the maximum walking distance for the experiment is limited. Using the tiles on floor, 6 parallel lines, each separated by 0.36 meter distance from another, are marked. The subject starts at one end and walks through the 1.8 meter distance with 5 steps, hitting the marked lines exactly. When the subject makes a mistake, such as missing the line, or step backwards, the trial ends and results are abandoned. Sometimes even if the subject performs perfectly, the trials can also produce bad results that have to be discarded. After inspecting these results it is found that counting extra steps can significantly inflate the total distance travelled. Further investigation shows that sometimes the step threshold $\mathrm{E}_{\mathrm{S}}$ is too small to contain the fluctuations in Euler angles, causing the system to count a non-existent step with a step length slightly greater than $E_{S}$. In some very rare cases, the system can miss a step when counting, therefore underestimating the total distance. Table 15 below shows the results of the experiment. In the first 3 trials, the subject starts stepping with left leg; in Trial 4 and Trial 5, the subject starts stepping with right leg. 
Table 15: MotionTracker experiment results with step by step measurements.

\begin{tabular}{|c|c|c|c|c|c|c|}
\hline \multicolumn{2}{|l|}{ Step } & 1 & 2 & 3 & 4 & 5 \\
\hline \multicolumn{2}{|c|}{ Actual Distance (m) } & 0.36 & 0.72 & 1.08 & 1.44 & 1.8 \\
\hline Trial 1 & Results (m) & 0.35 & 0.59 & 0.98 & 1.26 & 1.73 \\
\hline $\begin{array}{l}\text { Left leg } \\
\text { first }\end{array}$ & $\begin{array}{l}\text { Percentage } \\
\text { Errors }\end{array}$ & $-2.78 \%$ & $-18.06 \%$ & $-9.26 \%$ & $-12.50 \%$ & $-3.89 \%$ \\
\hline Trial 2 & Results (m) & 0.47 & 0.85 & 1.34 & 1.63 & 1.92 \\
\hline $\begin{array}{l}\text { Left leg } \\
\text { first }\end{array}$ & $\begin{array}{l}\text { Percentage } \\
\text { Errors }\end{array}$ & $30.56 \%$ & $18.06 \%$ & $24.07 \%$ & $13.19 \%$ & $6.67 \%$ \\
\hline Trial 3 & Results (m) & 0.37 & 0.89 & 1.2 & 1.48 & 1.83 \\
\hline $\begin{array}{l}\text { Left leg } \\
\text { first }\end{array}$ & $\begin{array}{l}\text { Percentage } \\
\text { Errors }\end{array}$ & $2.78 \%$ & $23.61 \%$ & $11.11 \%$ & $2.78 \%$ & $1.67 \%$ \\
\hline Trial 4 & Results (m) & 0.32 & 0.61 & 0.88 & 1.26 & 1.67 \\
\hline $\begin{array}{l}\text { Right leg } \\
\text { first }\end{array}$ & $\begin{array}{l}\text { Percentage } \\
\text { Errors }\end{array}$ & $-11.11 \%$ & $-15.28 \%$ & $-18.52 \%$ & $-12.50 \%$ & $-7.22 \%$ \\
\hline Trial 5 & Results (m) & 0.29 & 0.66 & 0.96 & 1.44 & 1.76 \\
\hline $\begin{array}{l}\text { Right leg } \\
\text { first }\end{array}$ & $\begin{array}{l}\text { Percentage } \\
\text { Errors }\end{array}$ & $-19.44 \%$ & $-8.33 \%$ & $-11.11 \%$ & $0.00 \%$ & $-2.22 \%$ \\
\hline
\end{tabular}

The results in Table 15 show that no matter which leg the subject starts stepping with, the final results after 5 steps are within 10\% range of the actual distance. In the first 3 steps there are some great discrepancy between measured distance and actual distance, but the difference narrows later in step 4 and 5 . This is because the inaccuracy in the model and the randomness of the legs' movements during a step magnifies the error when 
there are very few steps. The error becomes less significant in proportion as the number of steps and the total distance grow. Still it represents the primary error for the MotionTracker system. Better modelling, including more accurate numbers for the limb lengths and adjustments for the position of the sensors, could estimate these errors and improve the accuracy for the system. 


\section{Chapter 6: Conclusion and Future Work}

Inertial sensor networks have a huge potential for consumer-oriented applications, such as action capture, personal navigation, and consumer robotic control. As the MEMS sensors become increasingly accurate and the wireless communication becomes more efficient, inertial sensor networks are becoming an important supplement to mobile devices. The current inertial sensor networks are often designed for a specific application and thus lack the compatibility needed for consumer products. Therefore it is desired to have a low-cost open-source development platform which is compatible with most of today's consumer electronic devices.

\subsection{Conclusions}

The inertial sensor network developed in this thesis tries to fulfill this need by using low-cost sensors for measurements and Bluetooth Smart technology for transmission. Compared to other common wireless transmission protocols such as $\mathrm{Wi}-\mathrm{Fi}$ and classic Bluetooth, Bluetooth Smart technology consumes less power. Bluetooth Smart is also supported by most modern personal mobile computing devices such as smartphones, tablets, and laptop PCs. With limitations of unit price and physical size, the system aims to achieve reasonable accuracy, data rate, and battery life for a range of applications. Each sensor node of the prototype system takes input from a 9-axis MEMS sensor, which produces acceleration, angular velocity, and magnetic field measurements. With the sensor fusion algorithm, it is possible to calculate Euler angles, Quaternions, and rotation matrices real-time. Such a large set of data allow many possibilities in applications. The data are transmitted to a microcontroller on the sensor node board. This microcontroller is equipped with capabilities to do a large amount of floating point calculations real-time, allowing it to process the sensor results on the run, such as applying filters, removing biases, or estimating errors. This feature is essential since the bandwidth of the Bluetooth Smart is precious and reducing the amount of data for transmission can improve the maximum data rate. The processed and selected data are then sent over to the Bluetooth Smart module for transmission to the remote data processing device, where the data from all sensor nodes are synthesized and analyzed for applications. 
A simple personal navigation system is designed to demonstrate the application of the inertial sensor network. The concept of this PNS is based on measuring the tilt of the legs when walking, rather than integrating the accelerations. Four sensor nodes are placed just above and below knees of a user. An Android app is developed to collect data from all the sensor nodes and interface with the user. Once all the sensor nodes are connected, the app commands all nodes to start automatically transmitting Euler angles to the device when new values are measured. The app calculates step length from these angles when the two feet are furthest from each other. The step lengths are accumulated to produce the total distance travelled by the user. These results are displayed to the user during the process.

A series of experiments are conducted to test the performance of an individual sensor node. The results suggest that the sensor node produces good outputs for accelerometer and gyroscope when at rest. The Euler angle results calculated based the raw sensor inputs and the DCM library are also quite accurate. However the magnetometer of MPU9150 cannot produce measurements accurate enough to determine the orientation in home or office environment. When the sensor node is moving, the accuracy of the data drops. As the sensor node experiences large acceleration shock, the Euler angle could produce exaggerated results and only stabilize after a few seconds. The data rate of the sensor node is limited by the communication between the microcontroller and the BLE113 module. It is found that the communication between a sensor node and a Galaxy Nexus could work without missing data packets at a maximum frequency of $200 \mathrm{~Hz}$.

When there are 4 sensor nodes forming the inertial sensor network, the maximum data rate is limited by the remote data processing device. Using a Galaxy Nexus with Android system to receive the sensor node transmission, the maximum working frequency found in the experiment is $40 \mathrm{~Hz}$. This data rate should be good enough for many applications targeting consumer market.

An evaluation is also conducted for the MotionTracker example application. It is found that the distance estimated by the system has an error of roughly $10 \%$. 


\subsection{Future work on the inertial sensor network}

The inertial sensor network is designed to be an open-source development platform for consumer oriented applications. We hope developers of wearable sensor network applications can utilize this platform. Still the system could be improved in various ways to enable more potential applications on the platform.

First of all the sensor nodes need to have a working on-board battery system. The 3.3V rechargeable coin battery in hardware design is proved to be insufficient to power the whole sensor node alone. Potentially an additional same coin battery could solve the issue, but it would significantly increase the physical size of a sensor node. An ideal solution would be to replace the coin battery with a rechargeable battery pack. This type of battery pack could hold more energy than the coin battery and thus could last longer. A control circuit is needed to for recharging the battery pack when micro-USB cable is connected. The battery pack should be connected to the voltage regulator to output consistent $3.3 \mathrm{~V}$ voltage. Since the battery pack is rechargeable, it does not have to be exposed to allow replacements. Hence the battery could be removed from the surface of the board, reducing the physical size of the sensor node.

Another improvement could be done by further reducing the size of the sensor node board. As a prototype, all functionalities of the sensor node are currently placed on a single board for convenience. This has made the board unnecessarily large. As discussed in sub-section 3.1.5, the components could be placed on both sides of the board, and power-related components such as the regulator could be moved out of the main board. This way the sensor node could become two separate boards. The one with the sensor, microcontroller, and BLE module, has to be placed accurately for measurement; the other with the battery and power components can be placed anywhere that is comfortable for users.

Future developments of the system could first focus on improving the bottleneck that is limiting the performance of the system as a whole. The main bottleneck for the maximum data collection frequency is the remote data processing device that is receiving the data. Since the remote data processing device should be a consumer electronic device, 
its throughput improvements could come with the release of newer generations. On the other hand, if the amount of data required to transmit each cycle is reduced, the maximum data rate could increase. Developers of applications could try to use as few bytes of data as possible to transmit the results from sensor nodes to the remote data processing device.

Also, more complicated filters and error estimating algorithms could be deployed to the microcontroller of the sensor nodes. These algorithms should be application-specific with different assumptions for different scenarios. It is possible to significantly improve data quality with algorithms like Zero Velocity Update, Optimal bias estimation, and Kalman Filter.

As the MEMS technology improves, sensors with better accuracy and battery life could become available at a lower price. Inertial sensor network is going to see increasing penetration into everyday life of common people. The inertial sensor network prototype discussed in this thesis attempts to help the efforts of developing consumer-centered applications based on inertial sensors. The system could be updated with more accurate sensor and more efficient wireless communication protocol in the future, allowing a wider range of potential applications. 


\section{Reference}

[1] R. Nerino, L. Contin, W. J. Gonçalves da Silva Pinto, G. Massazza, M. Actis, P. Capacchione, A. Chimienti and G. Pettiti, "A BSN based service for post-surgical knee rehabilitation," in Proceedings of the 8th International Conference on Body Area Networks, Brussels, Belgium, Belgium, 2013.

[2] R. Slyper and J. K. Hodgins, "Action capture with accelerometers," in Proceedings of the 2008 ACM SIGGRAPH/Eurographics, Vienna, Austria, 2008.

[3] J. C. Alvarez, D. Alvarez, A. López and R. C. González, "Pedestrian navigation based on a waist-worn inertial sensor," Sensors, vol. 12, no. 8, pp. 10536-10549, 2012.

[4] R. Aylward and J. A. Paradiso, "A compact, high-speed, wearable sensor network for biomotion capture and interactive media," in Proceedings of the 6th international conference on Information processing in sensor networks, New York, NY, USA, 2007.

[5] R. Feliz, E. Zalama and J. Gómez, "Pedestrian tracking using inertial sensors," Journal of Physical Agents, vol. 3, no. 1, pp. 35-43, 2009.

[6] K. Abdulrahim, C. Hide, T. Moore and C. Hill, "Using constraints for shoe mounted indoor pedestrian navigation," Journal of Navigation, vol. 65, no. 1, pp. 15-28, 2012.

[7] J.-1. Zhang, Y.-y. Qin and C.-b. Mei, "Shoe-mounted personal navigation system based on," Journal of Chinese Inertial Technology, vol. 19, no. 3, pp. 253-256, 2011.

[8] "Wi-Fi Direct," [Online]. Available: http://www.wi-fi.org/discover-wi-fi/wi-fidirect. 
[9] "ZigBee Specification Overview," [Online]. Available: http://www.zigbee.org/Specifications/ZigBee/Overview.aspx.

[10] "A Look at the Basics of Bluetooth Technology," [Online]. Available: http://www.bluetooth.com/Pages/Basics.aspx.

[11] D. Vlasic, R. Adelsberger, G. Vannucci, J. Barnwell, M. Gross, W. Matusik and J. Popović, "Practical motion capture in everyday surroundings," ACM Transactions on Graphics, vol. 26, no. 3, p. 35, 2007.

[12] S. Y. Cho and C. G. Park, "MEMS based pedestrian navigation system," Journal of Navigation, vol. 59, no. 01, pp. 135-153, 2006.

[13] E. Foxlin, "Pedestrian tracking with shoe-mounted inertial sensors," IEEE Computer Graphics and Applications, vol. 25, no. 6, pp. 38-46, 2005.

[14] S. K. Park and Y. S. Suh, "A zero velocity detection algorithm using inertial sensors for pedestrian navigation systems," Sensors, vol. 10, no. 10, pp. 9163 9178, 2010.

[15] Ö. Bebek, M. A. Suster, S. Rajgopal, M. J. Fu, X. Huang, M. C. Cavusoglu, D. J. Young, M. Mehregany, A. J. V. D. Bogert and C. H. Mastrangelo, "Personal navigation via high-resolution gait-corrected inertial measurement units," IEEE Transactions on Instrumentation and Measurement, vol. 59, no. 11, pp. 30183027, 2010.

[16] R. Stirling, J. Collin, K. Fyfe and G. Lachapelle, "An innovative shoe-mounted pedestrian navigation system," in proceedings of European navigation conference GNSS, 2003.

[17] Texas Instruments, "Technical Documents for TM4C12x MCUs," 62014. [Online]. Available: http://www.ti.com/lit/pdf/spms376. 
[18] E. R. Bachmann, I. Duman, U. Y. Usta, R. B. McGhee, X. P. Yun and M. J. Zyda, "Orientation tracking for humans and robots using inertial sensors," Computational Intelligence in Robotics and Automation, 1999. CIRA'99. Proceedings. 1999 IEEE International Symposium on, pp. 187-194, 1999.

[19] R. Zhu and Z. Zhou, "A real-time articulated human motion tracking using tri-axis inertial/magnetic sensors package," Neural Systems and Rehabilitation Engineering, IEEE Transactions on, vol. 12, no. 2, pp. 295-302, 2004.

[20] Texas Instruments, "SensorTag," 2013. [Online]. Available: http://www.ti.com/ww/en/wireless_connectivity/sensortag/index.shtml?DCMP=se nsortag\&HQS=sensortag-bn. 


\section{Appendices}

\section{A. Cost of components for a sensor node}

The following table shows the list of components with costs, before tax and delivery fees. Pin headers and buttons are not specifically listed, instead, they are grouped together in "Other" category with an estimated cost of $\$ 5$ per sensor node. The total component cost of a sensor node before tax is $\$ 70.92$.

Table 16: Complete list of component costs of a sensor node

\begin{tabular}{|l|l|c|c|}
\hline Manufacture & Manufacture Part Number & Units & Costs (CAD) \\
\hline InvenSense & MPU9150 & 1 & $\$ 16.79$ \\
\hline MPD & BU2032SM-G & 1 & $\$ 0.95$ \\
\hline Taiyo Yuden & TMK212BJ474KD-T & 3 & $\$ 0.42$ \\
\hline TDK & C2012X5R2E222K085AA & 1 & $\$ 0.22$ \\
\hline TDK & C2012X5R2E103K125AA & 5 & $\$ 0.64$ \\
\hline TDK & C2012X5R1E105K125AA & 3 & $\$ 0.54$ \\
\hline Taiyo Yuden & TMK212BJ225KG-T & 1 & $\$ 0.24$ \\
\hline Johanson & 251R15S240JV4E & 2 & $\$ 0.78$ \\
\hline Kemet & C0805C100KBRACTU & 10.63 \\
\hline Hirose & PC62-AB-5PA(11) & $\$ 1.18$ \\
\hline NXP & Rageo & 2 & $\$ 0.16$ \\
\hline
\end{tabular}




\begin{tabular}{|l|l|c|c|}
\hline Yageo & RC0805JR-0710KL & 3 & $\$ 0.36$ \\
\hline Yageo & RC0805JR-07330RL & 4 & $\$ 0.09$ \\
\hline Panasonic & ERJ-6GEYJ472V & 2 & $\$ 0.24$ \\
\hline Yageo & RC0805FR-071ML & 1 & $\$ 0.12$ \\
\hline Texas Instruments & TPS73633DRBR & 1 & $\$ 2.66$ \\
\hline Abracon & AB26TRB-32.768KHZ-T & 1 & $\$ 0.62$ \\
\hline Texas Instruments & TM4C123GH6PMTR & 1 & $\$ 14.02$ \\
\hline Samsung & CL21F104ZBCNNNC & 1 & $\$ 21.76$ \\
\hline Abracon & ABM3-16.000MHZ-B2-T & $\$ 1.20$ \\
\hline Cree & CLVBA-FKA-CA1D181BB7R3R3 & 1 & $\$ 0.69$ \\
\hline C\&K Components & JS202011SCQN & 1 & $\$ 70.51$ \\
\hline Bluegiga & Other (buttons, pins headers) & 13 \\
\hline
\end{tabular}




\section{B. Source code for sensor node software}

The source code is hosted at Github, link:

https://github.com/rodericus1987/Sensor-Node-Software

The root folder contains the source files for TM4C123GH6PM microcontroller. These files need to be compiled with the software stack "Tivaware" provided by Texas Instruments, using Code Composer Studio (v5 or newer). The software tools and library could be downloaded from:

\section{http://www.ti.com/tool/sw-ek-tm4c123gxl}

To load the program, a TM4C Launchpad is needed. The Launchpad needs to connect debug out pins (TCK, TMS, TDO, TDI) with the sensor node, and then connects to PC with a microUSB wire. The sensor node should be powered and a common ground should be established between the sensor node and the Launchpad.

The "uart_demo" folder contains the configuration files for BLE113. These configuration files could only be compiled and updated to the Bluetooth Smart module via Bluegiga software tools and a Texas Instruments' CC debugger. 


\section{Source code for MotionTracker app}

The source code is also hosted at Github, link:

https://github.com/rodericus1987/MotionTrackerApp

The project should be edited and compiled using Eclipse IDE with Android SDK Tools. Follow the instructions below to load the program to an Android device:

a) Install the Eclipse ADT (with Android SDK Tools included) from http://developer.android.com/sdk/index.html

b) Download project file from Github, import it to the Eclipse workspace

c) Connect to an Android device with USB debugging enabled, run or debug the program 


\section{Schematics and PCB design files for the sensor node board}

The schematics and PCB design files can be found in the following link:

https://github.com/rodericus1987/Sensor-Node-Software/tree/master/schematics 